\title{
Photonic RF and microwave temporal signal processing based on optical Kerr micro-combs
}

\author{
Mengxi Tan, ${ }^{1}$ Xingyuan $\mathrm{Xu}^{2}{ }^{2}$ Jiayang $\mathrm{Wu},{ }^{1}$ and David J. Moss ${ }^{1}$
}

\author{
${ }^{1}$ Optical Sciences Centre, Swinburne University of Technology, Hawthorn, VIC 3122, Australia. \\ ${ }^{2}$ Electro-Photonics Laboratory, Dept. of Electrical and Computer Systems Engineering, Monash University, Australia.
}

\begin{abstract}
Integrated Kerr micro-combs are a powerful source of multiple wavelength channels for photonic radio frequency (RF) and microwave signal processing, particularly for transversal filter systems. They offer significant advantages featuring a compact device footprint, high versatility, large numbers of wavelengths, and wide Nyquist bands. We review progress photonic RF and microwave high bandwidth temporal signal processing based on Kerr micro-combs with comb spacings from $49 \mathrm{GHz}$ to $200 \mathrm{GHz}$. We focus on integral and fractional Hilbert transforms, differentiators as well as integrators. The future potential of optical micro-combs for RF photonic applications in terms of functionality and ability to realize integrated solutions is also discussed.
\end{abstract}

Keywords-Microwave photonics, micro-ring resonators, signal processing, microcombs, Kerr combs.

\section{INTRODUCTION}

All-optical signal processing based on nonlinear optics has proven to be extremely powerful, particularly when implemented in photonic integrated circuits based on highly nonlinear materials such as silicon [1-3]. All optical signal processing functions include all-optical logic [4], demultiplexing at ultra-high bit rates from 160Gb/s [5] to over 1Tb/s [6], optical performance monitoring (OPM) using slow light [7,8], all-optical regeneration [9,10], and others [11-16]. Complementary metal oxide semiconductor (CMOS) compatible platforms are centrosymmetric and so the $2^{\text {rd }}$ order nonlinear response is zero. Hence, nonlinear devices in these platforms have been based on the $3^{\text {rd }}$ order nonlinear susceptibility including third harmonic generation [11,17-21] and the Kerr nonlinearity $\left(n_{2}\right)$ [1,2]. The efficiency of Kerr nonlinearity based all-optical devices depends on the waveguide nonlinear parameter $(\gamma)$. Although silicon-on-insulator nanowire devices can achieve extremely high nonlinearities $(\gamma)$, they suffer from high nonlinear optical losses due to twophoton absorption (TPA) and the resulting generated free carriers [2]. Even if the free carriers are swept out by p-i-n junctions, silicon's relatively poor intrinsic nonlinear figure of merit $\left(\mathrm{FOM}=n_{2} /(\beta \lambda)\right.$, where $\beta$ is the TPA and $\lambda$ the wavelength) of around 0.3 in the telecom band is too low to achieve good performance. While TPA can be turned to advantage for some all-optical functions [22-24], for the most part, silicon's low FOM in the telecom band is a limitation. This has motivated research on a range of alternate nonlinear platforms including chalcogenide glasses [25-34]. However, while offering many advantages, these platforms are not compatible with CMOS processing - the basis of the silicon computer chip industry.

In 2007/8 new CMOS compatible platforms for nonlinear optics were reported that exhibited extremely low two-photon absorption in the telecommunications wavelength band. These included silicon nitride $[35,36]$ as well as high-index doped silica glass (Hydex) [37-47], similar in its optical properties to silicon oxynitride. In addition to negligible nonlinear absorption, these platforms displayed a moderate Kerr nonlinearity, resulting in an extremely high nonlinear figure of merit as well as a nonlinear parameter that was high enough to support substantial parametric gain. Following the first report of micro-ring resonator (MRR) based frequency comb source driven by the Kerr optical nonlinearity in 2007 [48], the first fully integrated optical parametric oscillators were reported in $2010[36,37]$ that were also CMOS compatible. Since then the field of integrated micro-combs, or "Kerr combs" has become one of the largest fields in optics and photonics [47]. Integrated optical Kerr frequency comb sources, or "micro-combs" are a fundamentally new and powerful tool to accomplish many new functions on an integrated chip, due to their ability to provide highly coherent combs of multiple wavelength channels, at the same time offering a very high degree of wavelength spacing control. Optical micro-combs are produced via optical parametric oscillation driven by modulational instability gain in monolithic MRRs. They offer significant advantages over more traditional multi-wavelength sources. Many innovative applications have been reported based on integrated Kerr micro-combs, from filter-driven mode-locked lasers [49-52] to quantum optical photonic chips [53-61], ultrahigh bandwidth optical fiber data transmission [62-64], optical neural networks [65-67], integrated optical frequency synthesizers [68]. Micro-combs have been extensively reviewed [47, 69 - 76]. The success of the new CMOS 
platforms silicon nitride and Hydex motivated the search for even higher performing CMOS compatible platforms such as amorphous silicon [77] and silicon rich silicon nitride [78] that display a combination of low linear loss, high nonlinearity and very low nonlinear loss.

All-optical signal processing based on nonlinear optics has attracted significant interest over the years for its ability to achieve ultrahigh bandwidth without needing optical to electronic (or visa versa) conversion. It has been particularly important for a wide range of signal processing functions for both telecommunications and RF/microwave applications. RF photonic applications range from radar systems to signal generation and processing [79-128], and are attractive because of the ultra-high bandwidths that can be achieved as well as low transmission loss and strong electromagnetic interference immunity. There is a very wide range photonic RF approaches including techniques that map the optical filter response in the optical domain onto the RF domain. This is perhaps best represented by integrated devices based on stimulated Brillouin scattering [88-95], which has resulted in extremely high performance for RF resolution — being able to resolve features as fine as $32 \mathrm{MHz}$, at the same time achieving a stopband rejection > $55 \mathrm{~dB}$. A key approach to achieving reconfigurable transfer functions for adaptive signal processing has been based on the transversal filter method [96-100] that operate by generating weighted, progressively delayed replicas of an RF signal imprinted on an optical carrier and then summing them via photo-detection. Transverse filters can realize a wide range of RF signal processing functions by only adjusting the tap weights, and so this method is very attractive to realize advanced RF filters that need to be dynamically adaptive. Both discrete laser diode arrays [101] as well as integrated and fibre Bragg grating arrays and sampled gratings [103] have been successful at supplying the required taps. However, while offering many advantages, these approaches suffer from significantly increased complexity as well as reduced performance due to limitations in the available number of taps. Alternative approaches, such as employing optical frequency comb sources achieved by electro-optic (EO) or acoustooptic (AO) modulation [102,104,105], can help overcome this problem, but they require multiple high frequency
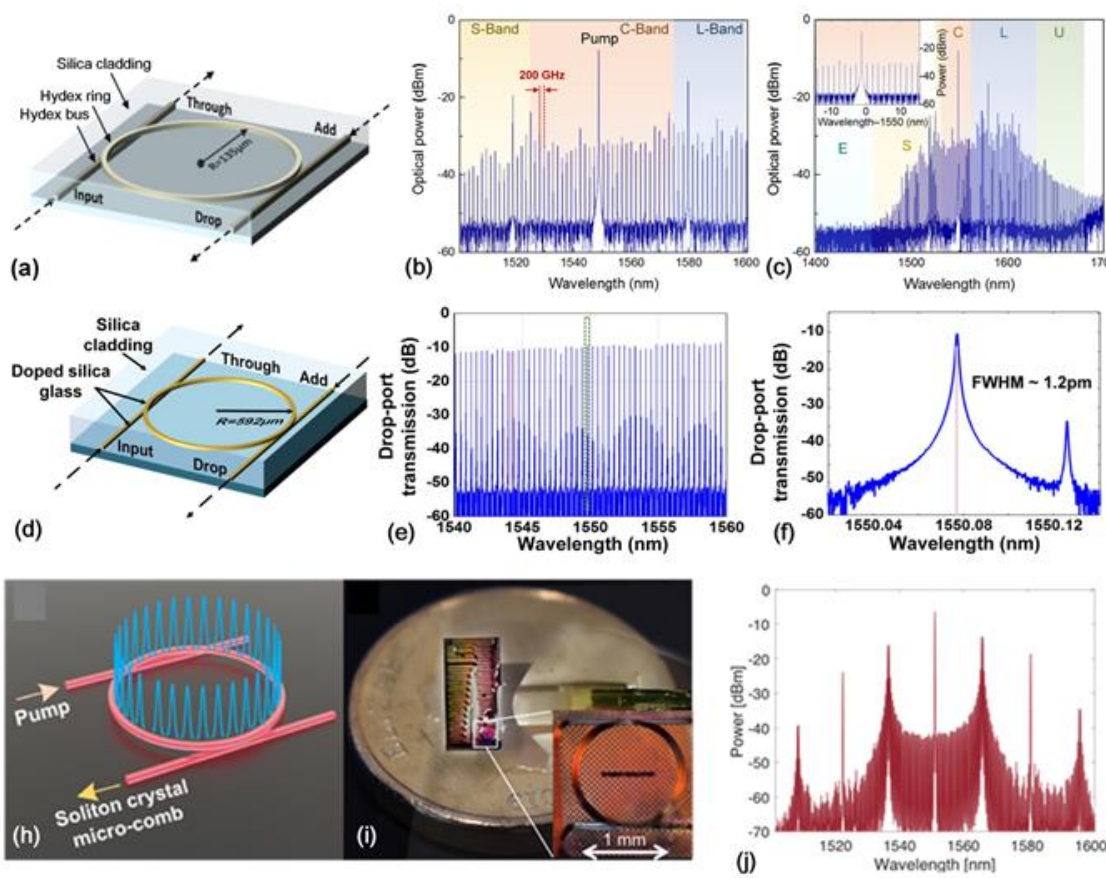

Fig. 1. Illustration of the integrated MRRs for generating the Kerr micro-comb for (a-c) $200 \mathrm{GHz}$ FSR combs and (d-j) 49GHz combs. (b, c) Optical spectra of the micro-combs generated by $200 \mathrm{GHz}$ MRR with a span of (b) $100 \mathrm{~nm}$ and (c) $300 \mathrm{~nm}$. (j) Optical spectra of the micro-combs generated by $50 \mathrm{GHz}$ MRR with a span of $100 \mathrm{~nm}$. (e) Drop-port transmission spectrum of the integrated MRR with a span of $5 \mathrm{~nm}$, showing an opticalfree spectral range of $49 \mathrm{GHz}$. (f) A resonance at $193.294 \mathrm{THz}$ with FWHM) of $124.94 \mathrm{MHz}$ corresponding to a $\mathrm{Q}$ of $1.549 \times 10^{6}$. have much wider spacings from 10's to 100's of GHz and even into the THz regime. Larger comb spacings yield much frequency RF sources. Of these approaches, EO combs have been used the longest and have achieved significant success at realizing many powerful functions.

Integrated optical Kerr microcombs are a relatively recent innovation and have been very successful - offering many advantages over other approaches that use multi-wavelength sources for RF applications. They have been demonstrated to be capable of achieving extremely high bit rate telecommunications systems as well as a very wide range of $\mathrm{RF}$ signal processing functions [107-128]. They can generate frequency combs with a much larger spacing than electrooptic combs. EO combs and micro-combs are in fact complementary in many ways EO combs focuses on finer wavelength spacings from 10's of $\mathrm{MHz}$ to $10-20 \mathrm{GHz}$, while integrated micro-combs typically modulators that rely on high- 
wider Nyquist zones that are needed to achieve large RF bandwidths, whereas finer spacings provide much larger numbers of wavelengths or RF "taps", but at the cost of a much smaller Nyquist zone, or RF bandwidth. Micro-combs can provide higher numbers of wavelengths together with still being able to provide a large FSR, in a compact footprint. For RF transversal filters the number of taps, or wavelengths, dictates the available number of channels for, for example, RF true time delays as well as determining the performance of RF filters [85, 121]. Other systems such as beamforming devices [112] can also be greatly improved in terms of their quality factor and angular resolution. Other innovative approaches to filtering include techniques such as RF bandwidth scaling [125] that provide a certain bandwidth for each wavelength channel, and so the total operation bandwidth (maximum RF signal bandwidth that can be processed) will depend on the number of wavelengths, and will therefore be significantly enhanced by the use of micro-combs.

Recently [121], we reviewed transversal filtering and bandwidth scaling methods based on Kerr micro-combs applied to RF and microwave spectral filters. Here, we focus on RF and microwave high bandwidth temporal signal processing based on integrated Kerr micro-comb devices. We review both integral and fractional order Hilbert transformers and differentiators, as well as RF integrators. We discuss the trade-offs involved between using wide spaced micro-combs with an FSR of 200GHz [109-111] with recently reported record low FSR spaced micro-combs at $49 \mathrm{GHz}$, based on soliton crystals [122-128]. We highlight their potential and future possibilities, contrasting the different methods and use of the differently spaced micro-combs. While $200 \mathrm{GHz}$ Kerr micro-combs have proven to be a powerful source for RF transversal filters, enabling high versatility as well as dynamic reconfigurability, the relatively large comb spacing FSR of $\sim 1.6 \mathrm{~nm}$ restricts the number of taps to typically less than 21 wavelengths within the $30 \mathrm{~nm}$ wide $\mathrm{C}$-band. This is important since transversal filters require optical amplifiers and spectral shapers that are typically only available in the telecommunications bands (1530-1620nm). This limitation in the tap number has restricted the performance of micro-comb based transversal RF filters in frequency selectivity, bandwidth, and dynamic versatility. To improve on this, we reported micro-comb-based photonic RF transversal filters operating with a record high number of taps - up to 80 wavelengths over the C-band [117]. This is the highest number reported for micro-comb RF transversal filters, and has been enabled by a record low $49 \mathrm{GHz}$ FSR integrated Kerr micro-comb source. For filter applications [121] this enabled $Q_{\mathrm{RF}}$ factors for RF bandpass filters 4 times higher than that achievable with $200 \mathrm{GHz}$ combs. For temporal signal processing, this yields dramatic improvements in both bandwidth and reconfigurability. Our results confirm the feasibility of achieving high performance advanced as well as adaptive RF transversal filters for use in high bandwidth RF signal processing systems, at the same time providing reduced cost, footprint, and complexity.

\section{KERR MICROCOMBS}

The generation of micro-combs is a complex process that relies on a combination of high nonlinear optical parameters,
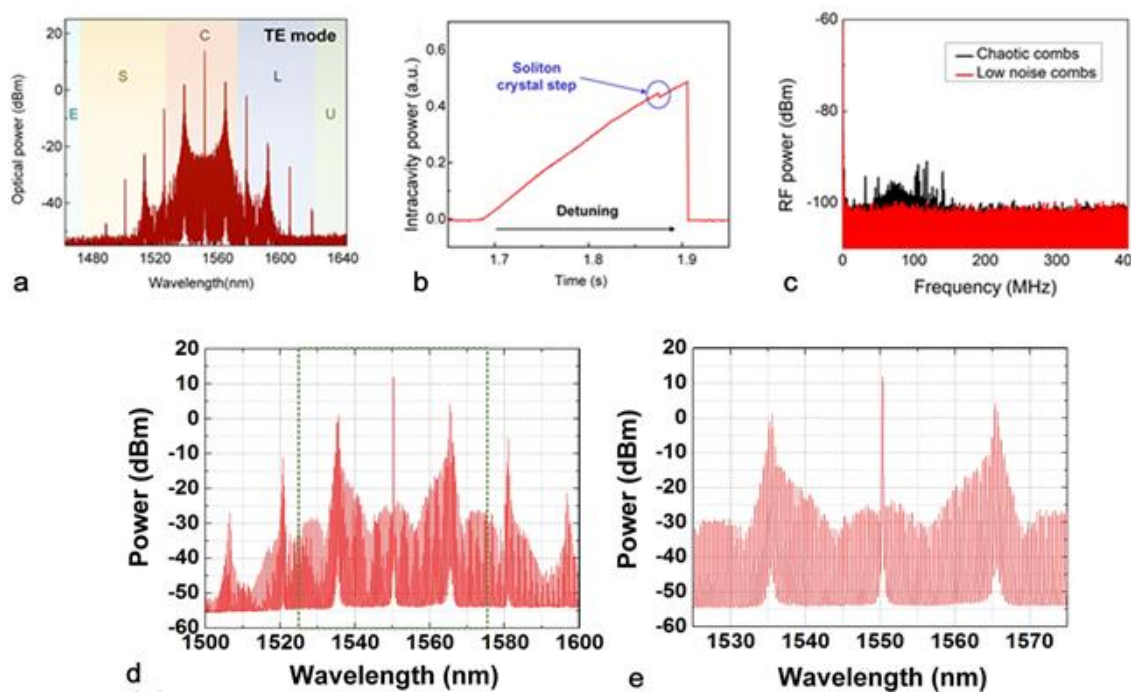

Fig. 2. (a, d, e) Optical spectra of soliton crystal micro-combs. (b) Optical power output vs pump tuning, showing the very small power jump at the onset of soliton crystal combs. (c) Transition from high RF noise chaotic state to low noise soliton crvstal comb state. low linear and nonlinear loss as well as engineered dispersion. Diverse platforms have been developed for micro-combs [47] such as silica, magnesium fluoride, silicon nitride, and doped silica glass [47, 70, 85]. In 2008 [39] we reported efficient four-wave mixing at milliwatt power levels based on the $575 \mathrm{GHz}$ FSR devices that had a relatively low Q-factor of 60,000 . This represented the first report of low power $\mathrm{CW}$ nonlinear optics in silica glass based MRRs. This was followed in 2010 by the first integrated microcombs [37, 38] in Hydex and silicon nitride - the first optical micro-combs that were realized in an integrated photonic chip, that 

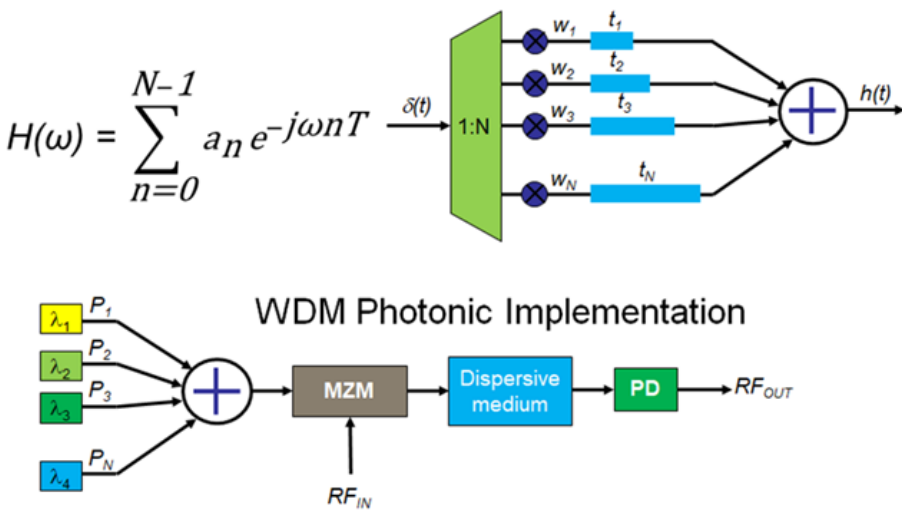

were inspired by the micro-combs demonstrated in toroidal resonators [48]. Another breakthrough came with the report of $[116,117]$ integrated micro-combs with record low spacings of $<50 \mathrm{GHz}$ which greatly expanded the number of wavelengths over the telecommunications band to 80 or more. Apart from a record low FSR, these micro-combs operated via a different process to single soliton states [66-73], called soliton crystals $[129,130]$. Many other recent breakthroughs have been reported in micro-combs, such as ultralow pump power combs [131], dark solitons [132], laser-cavity solitons [133] and others [134-139].

Fig. 3. Theoretical schematic of the principle of transversal filters using The MRRs used in the work that is reviewed here wavelength multiplexing. MZM: Mach-Zehnder modulators. PD: photo- were fabricated on a platform based on Hydex glass detector.

$[37,38]$ using CMOS compatible fabrication processes. Ring resonators with $\mathrm{Q}$ factors ranging from 60,000 to $>1.5$ million and with radii ranging from $\sim 592 \mu \mathrm{m}$ to $\sim 135 \mu \mathrm{m}$ and even smaller at $\sim 48 \mu \mathrm{m}$, corresponding to FSRs of $\sim 0.4 \mathrm{~nm}(\sim 49 \mathrm{GHz}), \sim 1.6 \mathrm{~nm}(\sim 200 \mathrm{GHz})$, and $\sim 4.5 \mathrm{~nm}$ $(\sim 575 \mathrm{GHz})$, respectively. The RF signal processing devices that are reviewed here were based on micro-combs with FSR spacings of $200 \mathrm{GHz}$ (Fig. 1a) and $49 \mathrm{GHz}$ (Fig. 1b). Hydex glass films $(n=\sim 1.7$ at $1550 \mathrm{~nm}$ ) were deposited via plasma enhanced chemical vapour deposition, and then patterned by deep UV stepper based photolithography. The waveguides were etched by inductively coupled plasma reactive ion etching to achieve waveguides with very low surface roughness. Finally, an upper cladding layer composed of silica glass $(n=\sim 1.44$ at $1550 \mathrm{~nm}$ ) was deposited. We typically a vertical
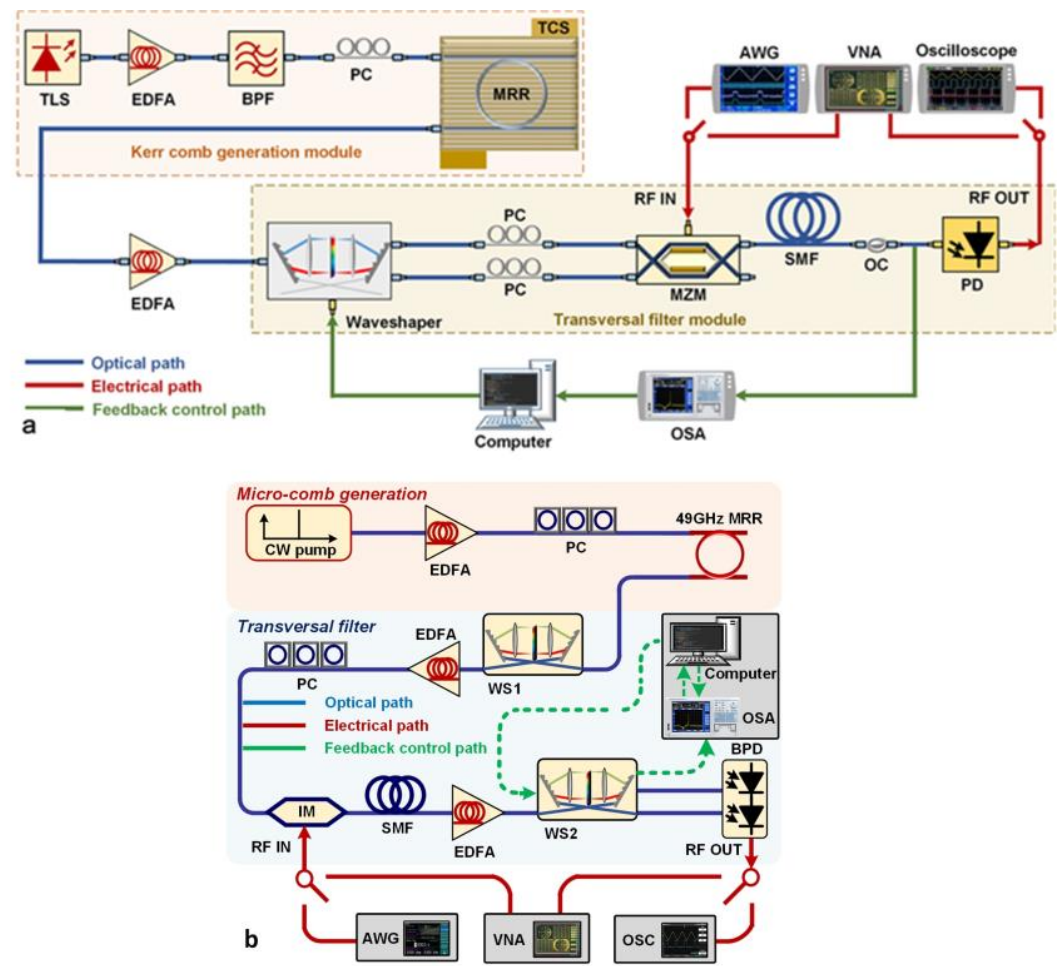

Fig. 4. RF transversal filters based on $200 \mathrm{GHz}$ microcomb (top) and $49 \mathrm{GHz}$ microcomb (bottom). TLS: tunable laser source. EDFA: erbium-doped fiber amplifier. PC: polarization controller. BPF: optical bandpass filter. TCS: temperature control (MR: micro-ring resonator. WS:WaveShaper. OC:optical coupler. SMF: single mode fibre. OSA: optical spectrum analyzer. AWG: arbitrary waveform generator. between the pump wavelength and MRR's VNA: vector network analyser. PD: photodetector. BPD: balanced photodetector. coupling scheme between the bus and ring resonator, where the gap of about $200 \mathrm{~nm}$ can be controlled by film growth, that is much more accurate than lithography. The advantages of the Hydex platform, particularly with respect to optical microcombs, include its very low linear loss $\left(\sim 0.06 \mathrm{~dB} \cdot \mathrm{cm}^{-1}\right)$, its moderately high optical nonlinear parameter $(\sim 233$ $\mathrm{W}^{-1} \cdot \mathrm{km}^{-1}$ ), and lastly, in particular its negligible nonlinear optical loss, even up to extremely high intensities of 25 $\mathrm{GW} \cdot \mathrm{cm}^{-2}$. We reported narrow resonance linewidths corresponding to $\mathrm{Q}$ factors of up to $\sim 1.5$ million (Fig. 1) for both the $49 \mathrm{GHz}$ and $200 \mathrm{GHz}$ FSR MRRs. After packaging the devices with fiber pigtails, the through-port insertion loss was typically 0.5 to $1 \mathrm{~dB}$ per facet - a result of very efficient on-chip mode converters.

To generate micro-combs with the $200 \mathrm{GHz}$ FSR devices, the continuouswave $(\mathrm{CW})$ pump power was typically amplified to over $\sim+30 \mathrm{dBm}$ and then the wavelength was swept from blue to red near one of the TE resonances near $\sim 1550$ cold resonant wavelength became small 
enough, thus allowing the intracavity power to reach a threshold, modulation instability gain induced oscillation occurred [47]. Primary combs were generated with the spacing determined by the MI gain peak - mainly a function of the intracavity power and dispersion. As the detuning was changed further, finally single-FSR spaced micro-combs eventually appeared. While the states that were reached by the micro-combs did not display soliton behaviour, we note that achieving rigorous single soliton states such as the dissipative Kerr solitons (DKS) [66] is not required. This is significant since, while much is now understood about DKS solitons and significant recent progress has been made [139], they still require complex pump dynamics involving simultaneous frequency and amplitude sweeping in both directions in order to "capture" or "kick" the soliton states out of the chaotic state. Our initial work on micro-comb applications was based on the $200 \mathrm{GHz}$ micro-combs operating in this partially coherent state that, while not exhibiting rigorous soliton behaviour, was nonetheless a relatively low noise state that managed to avoid the chaotic regime [47] and which we found was adequate for microwave applications. The spectra shown in Figs. $1 \mathrm{~b}$ and $1 \mathrm{c}$ are typical of the $200 \mathrm{GHz}$ combs in this initial microwave work, and clearly show that they are not a rigorous soliton states. They were successful at demonstrating a wide range of RF signal processing functions [85, 107, 109 -111].

Subsequently, we reported Kerr solitons with a record low FSR of 49GHz, based on a new class of soliton [129, 130] called soliton crystals. Soliton crystals result from mode crossings, in our case in the C-band, and are easier and more robust to generate than DKS states, and indeed even easier than the partially coherent $200 \mathrm{GHz}$ FSR comb states. They can be generated even with manual tuning of the pump laser wavelength. The underlying physics behind this is that the internal cavity optical energy of the soliton crystal state is very close to that of the chaotic state. Therefore, when transitioning from chaos to the soliton crystal state, there is only a very small shift in internal energy and hence very little resonant wavelength jump. It is this "jump" in both internal energy and resonant wavelengths of the micro-resonator that makes DKS states more challenging to achieve. At the same time, this same issue makes the efficiency of the soliton crystal states (energy in the comb lines relative to the pump wavelength) much higher than DKS states (the single soliton states in particular). There is a trade-off with soliton crystal microcombs, though, in that their spectra are not flat but exhibit characteristic "curtainlike" patterns. While this can sometimes require spectral flattening, it has not posed a fundamental barrier to achieving a wide range of high performance RF and microwave processing functions. Soliton crystals also require mode-crossings in the resonator, and this can be somewhat challenging to design, over and above the requirement of anomalous dispersion that DKS states need. This, however, has not posed a barrier to fabricating many chips with a high yield [64].

When generating soliton crystals, we typically tuned the pump laser wavelength manually across a resonance (TE polarized). When the pump (with sufficient power) was aligned well enough with the resonance, a primary comb was generated, similar to the $200 \mathrm{GHz}$ states. In this case, however, as the detuning was changed further, distinctive 'fingerprint' optical spectra were observed (Fig. 2), arising from spectral interference between tightly packed solitons in the cavity solitons that formed the soliton crystals $[129,130]$. A small but noticeable and abrupt step in the measured intracavity power (Fig. 2(b)) was observed at the point where these spectra appeared. More dramatic was a a very significant reduction in the RF intensity noise (Fig. 2(c)) at this point. Together with the spectra shapes, these observations indicate soliton crystal formation [38], although to conclusively demonstrate this would need one to perform time-resolved autocorrelation pulse measurements. The key issue for the RF experiments was not the specific micro-comb state that was reached, but whether or not low RF noise and high coherence states were achieved, and we found that these were relatively straightforward to achieve through adiabatic pump wavelength sweeping. We found that overall soliton crystals provided by far the lowest noise states of all micro-combs, and have formed the basis for a microwave oscillator with low phasenoise [120].

\section{RF TRANSVERSAL FILTER: THEORY AND EXPERIMENT}

The transfer function of a general transversal structure can be described as [85]

$$
F(\omega)=\sum_{k=0}^{M-1} h_{k} e^{-j \omega K T}
$$

(1)

Where $j=\sqrt{-1}, \omega$ is the angular frequency of the input RF signal, $M$ is the number of taps, $T$ is the time delay between adjacent taps, and $h_{k}$ is the tap coefficient of the $k_{t h}$ tap, which is the discrete impulse response of $F(\omega)$. The discrete impulse response can be calculated by performing the inverse Fourier transform of $F(\omega)$, and then temporally windowing it with a short cosine bell. The transversal structure is essentially equivalent to a finite impulse response digital filter, and both are 

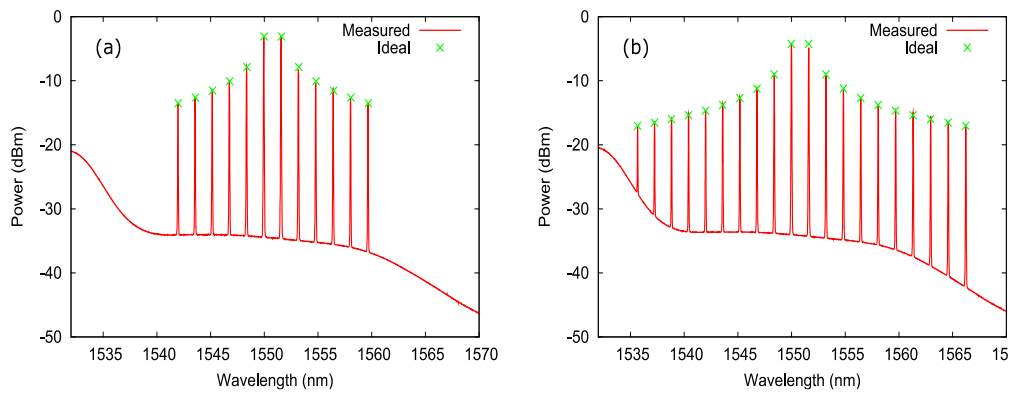

Fig. 5. Shaped optical spectra showing the weight of each tap for: (a) fundamental tools for RF signal processing in general and photonic RF signal processing in particular. By properly designing the tap weights $h_{k}$ for each wavelength channel, the transfer function for different signal processing functions can be realized, including Hilbert transforms, differentiators and integrators. The Nyquist frequency of the response function, which determines the maximum RF frequency that the devices can operate at, is given by $f_{\text {Nyquist }}$ $=1 / 2 T$. Figure 3 shows the schematic of a

transversal filter based on a multi-wavelength source, showing that the principle consists of several functions: First, all of the wavelengths from the microcomb source are imprinted with the RF signal via an electro-optic modulator - essentially broadcasting, or multicasting, the RF signal onto all the comb lines. This is then followed by transmitting the wavelengths through a dispersive medium to acquire wavelength-dependent delays. Second-order dispersion, which yields a linear relationship between the wavelength and the delay, is generally employed to progressively separate the replicas. The delayed signals can be either separately converted into electronic RF signals or summed together upon photodetection. Separately converting the signals can be achieved with wavelength demultiplexers and photodetector arrays for applications such as RF true time delays [116]. Summing the signals on all of the wavelengths forms the basis for RF transversal filters for signal processing functions [85]. The large number of wavelengths supplied by the micro-combs has significant advantages for enhancing the transversal signal processor performance. The number and amplitude of progressively delayed RF replicas, or taps, determines the performance of the system. By setting the tap coefficients, a reconfigurable transversal filter with virtually any transfer function can be achieved, including both integral and fractional order Hilbert transformers [107, 126], integral and fractional differentiators [109,127], bandpass filters [111,117, 121], integrators [123], waveform generators [128] and much more.

Figure 4 shows the experimental setup for a transversal filter based on an integrated Kerr optical comb source with an FSR of $200 \mathrm{GHz}$ (Fig. 4(a)) as well as the setup based on a 49GHz (Fig. 4(b)) comb. In both cases the micro-combs, generated by the on-chip MRR, are amplified and fed to either one (200GHz comb) or two (49GHz comb) waveshapers for channel equalization and weighting. A $2^{\text {nd }}$ waveshaper was used for the $49 \mathrm{GHz}$ FSR comb to pre-flatten the nonuniform comb spectra of the soliton crystals. The power of each comb line is then set by the waveshaper according to the required tap weights. To increase the accuracy, real-time feedback control paths are used to read and shape the power of the comb lines. The comb lines are then spatially divided into two paths according to the sign of the tap coefficients - one path for negative weights and one for positive. Next, for the $200 \mathrm{GHz}$ micro-comb experiments the signal was passed through a $2 \times 2$ balanced Mach-Zehnder modulator (MZM) that was biased at quadrature. The $2 \times 2$ balanced MZM simultaneously modulated the input RF signal on both positive and negative slopes, thus yielding replicas of the input RF signal with phase and tap coefficients having both algebraic signs. For experiments with the 49GHz FSR microcomb, both positive and negative taps were achieved by spatially separating the wavelength channels according to the designed tap coefficients and then feeding them into the respective positive and negative input ports of a balanced photodetector (Finisar BPDV2150R) (Figure 4b).
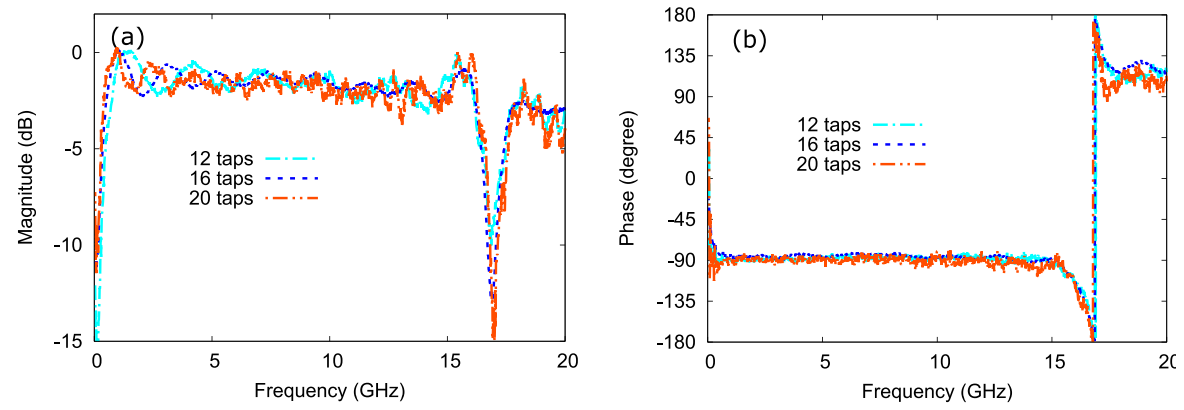

Fig. 6. Measured system RF frequency response for different number of filter taps: (a) amplitude; and (b) phase response.
For the system based on the $200 \mathrm{GHz}$ comb, the signal that was modulated by the MZM then went through $\sim 2.122-\mathrm{km}$ of standard single mode fibre (SMF), where the dispersion was $\quad 17.4$ $\mathrm{ps} /(\mathrm{nm} \cdot \mathrm{km})$, corresponding to a time delay $T$ of $\sim 59 \mathrm{ps}$ between adjacent wavelengths, or taps (the channel spacing of the time delay lines equalled the FSR 
of the micro-combs), which resulted in a Nyquist frequency of $\sim 8.45 \mathrm{GHz}$. The bandwidth of the systems was determined by the Nyquist frequency, which could be easily enlarged by decreasing the time delay which, because of the large FSR of the compact MRR, could reach over $100 \mathrm{GHz}$. Finally, the weighted and delayed taps were combined upon photodetection with a high-speed photodetector (Finisar, $40 \mathrm{GHz}$ bandwidth) which converted them back into RF signals.

For the transversal system based on the 49GHz FSR microcomb, the signal went through about $5 \mathrm{~km}$ of standard SMF to provide the progressive delay taps. The dispersion of the fibre was the same $(17 \mathrm{ps} / \mathrm{nm} / \mathrm{km})$ as the $200 \mathrm{GHz}$ system, which yielded a different time delay $T$ of $\sim 34.8$ ps between adjacent taps. This yielded an operational bandwidth (Nyquist frequency, half of $F S R_{\mathrm{RF}}$ ) of about $14.36 \mathrm{GHz}$ for the transversal filter. As before, this can be increased by decreasing the time delay (using a shorter spool of SMF), at the expense of having a reduced resolution in tuning. The maximum operational bandwidth of the transversal filter is limited by the comb spacing. For the $49 \mathrm{GHz}$ comb, significant crosstalk between adjacent wavelength channels occurs for RF frequencies that are beyond $24.5 \mathrm{GHz}$ - half of the micro-comb's spacing. This issue can be addressed by employing a micro-comb source with a larger comb spacing, although this comes at the expense of providing fewer comb lines/taps across the C-band. Note that although standard optical fibre is often used to produce the dispersive delays, this can readily be done much more efficiently using other approaches. Typically, only about $2-\mathrm{km}$ of SMF, yielding a dispersion of $34 \mathrm{ps} / \mathrm{nm}$, is needed. This is within the range of multi-channel tunable dispersion compensators [140-143], which can also be designed with a built-in dispersion slope offset. This approach would not only yield a compact and latency free delay but would enable tunability to adjust the Nyquist zone. In the following sections, we will review progress made in high bandwidth temporal RF and microwave signal processing functions beginning with Hilbert transforms, followed by differentiation and then integration, all based on Kerr microcombs.

\section{HILBERT TRANSFORMS}

The Hilbert transform (HT) is a fundamental signal processing operation with wide applications in radar systems, measurement systems, speech processing, signal sampling, single sideband modulators, and others [144]. Standard integral HTs perform a $\pm 90^{\circ}$ phase shift around a central frequency, with an all-pass amplitude transmission. Fractional Hilbert transforms (FHTs) that yield a variable phase shift represent a powerful extension to the standard HT, with applications to secure single sideband communications [145], hardware keys [145, 146], and in forming images that are edge enhanced

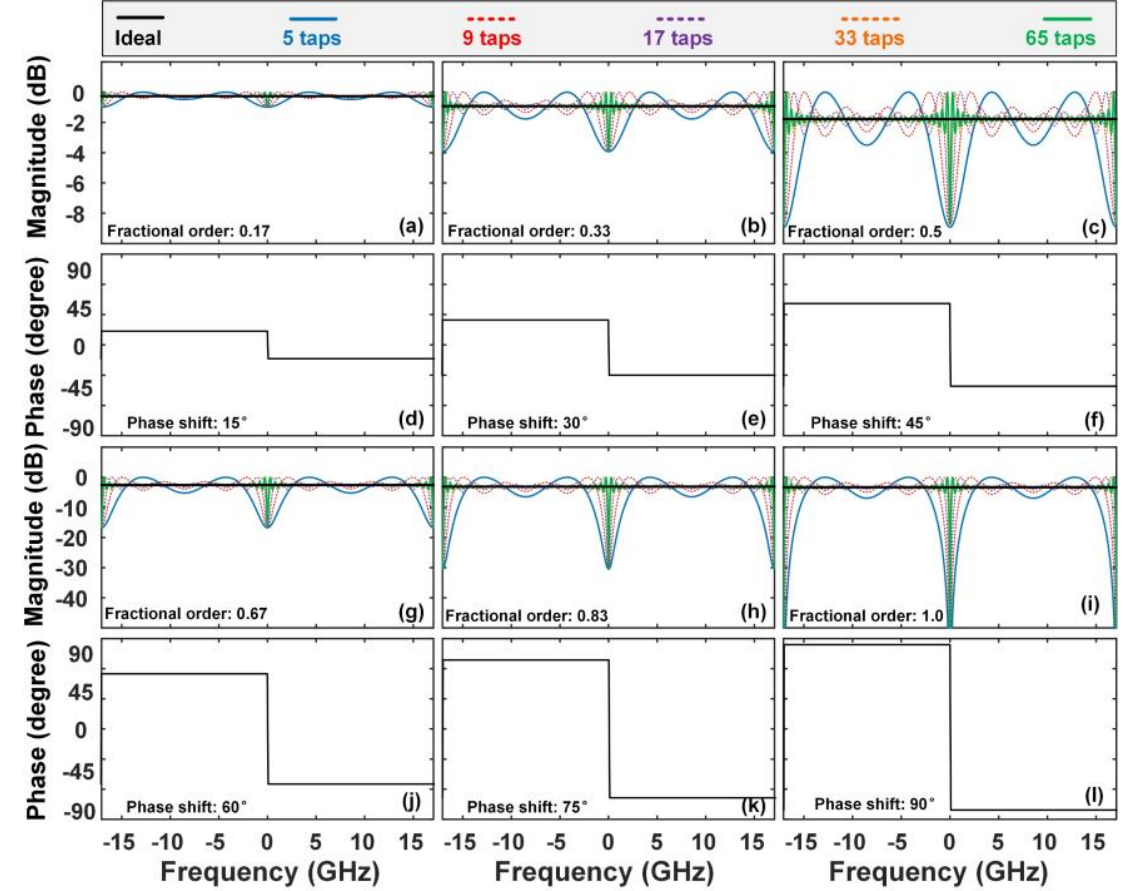

Fig. 7. Theoretical RF amplitude and phase response of FHTs with (a, d) $15^{\circ}$, (b, e) $30^{\circ}$, (c, f) $45^{\circ},(\mathrm{g}, \mathrm{j}) 60^{\circ}$, (h, k) $75^{\circ}$, and $(\mathrm{i}, \mathrm{l}) 90^{\circ}$ phase shifts. The amplitude of the fractional Hilbert transformers designed based on Eq. (3) (colour curves) are shown according to the number of taps employed. relative to the input object, where one can select the edges that are enhanced as well as the degree of edge enhancement [147]. Electronic fractional Hilbert transformers are limited in operation bandwidth [145, 148], whereas photonic technologies offer broadband operation as well as strong immunity to EMI. Hilbert transformers based on free-space optics have been demonstrated [146, 149] that achieve high performance but are bulky and complex. Phaseshifted fibre Bragg grating based Hilbert transformers typically have bandwidths of a few 100GHz [150153] but only yield a precise FHT for signals with specific bandwidths, fixed fractional orders, and only operate on the complex optical field (not the actual RF signal). This also holds for integrated reconfigurable microwave processors [154] as well 
Bragg grating approaches [155]. In practice, the FHT of the RF and microwave signals - not the complex optical fields is what is most desired for RF measurement and signal reshaping [156-163].

Integrated Hilbert transformers realized using micro-ring or micro-disc resonators, Bragg gratings in silicon, and integrated InP-InGaAsP photonic chips yield compact devices with high stability and mass-producibility [154, 155, 159161]. Nevertheless, they only operate on the complex optical field and generally only provide a limited phase shift tuning range. Transversal filter approaches offer high reconfigurability [162, 163] but need many discrete lasers, thus increasing system size, cost, and complexity, and limiting the number of taps and therefore performance. Optical frequency combs (OFCs) provide a single high-quality source for many wavelengths, including mode-locked lasers [164], electro-optical modulators [165], and micro-resonators [166]. Of these, Kerr micro-combs generated by micro-resonators provide many wavelengths with greatly reduced footprint.

The spectral transfer function of a general fractional Hilbert transformer can be expressed as [80, 126]:

$$
H_{P}(\omega)=\left\{\begin{array}{c}
e^{-j \varphi}, \text { if } 0 \leq \omega<\pi \\
e^{j \varphi}, \text { if }-\pi \leq \omega<0
\end{array}\right.
$$

where $j=\sqrt{-1}, \varphi=P \times \pi / 2$ is the phase shift, and $P$ denotes the fractional order. From Eq. (2), a FHT can be regarded as a phase shifter with $\pm \varphi$ phase shifts centered around a frequency $\omega$, which becomes a classical integral HT when $P=1$. The corresponding impulse response is a continuous hyperbolic function:

$$
h_{P}(t)=\left\{\begin{array}{c}
\frac{1}{\pi t}, t \neq 0 \\
\cot (\varphi), t=0
\end{array}\right.
$$

For digital implementations, this hyperbolic function is truncated and sampled in time by discrete taps. The sample spacing $\Delta t$ determines the null frequency $f_{\mathrm{c}}=1 / \Delta t$. The order of the FHT is continuously tunable by only adjusting the coefficient of the tap at $t=0$ while keeping the same coefficients for the other taps [146].

To achieve a Hilbert Transform the normalized power of each comb line is given by:

$$
p_{n}=\frac{1}{\pi|n-N / 2+0.5|}
$$

where $N$ is the number of comb lines, or filter taps, used in the filters, and $n=0,1,2, \ldots, N-1$ is the comb index.

In 2015, the first Kerr micro-comb based Hilbert transformers was demonstrated, with up to 20 taps [107] from a 200GHz comb that achieved a record RF bandwidth (5 octaves). Figures 5(a) and 5(b) show the shaped optical micro-combs measured using an optical spectrum analyzer for the 12 and 20 tap filter cases, respectively. The designed tap weights, or wavelength target powers, are also shown as green crosses in Figs. 5(a) and 5(b). The waveshaper was used to shape the powers of all comb lines successfully to within $+/-0.5 \mathrm{~dB}$ of the target powers. This could easily be achieved since the waveshaper resolution of $10 \mathrm{GHz}$ was much smaller than the comb spacing which was $200 \mathrm{GHz}$ at the time. Once the comb lines were attenuated in order to provide the correct tap coefficients of the impulse response associated with a HT, with the unused comb lines attenuated below the noise floor, the system RF frequency response both in amplitude and phase was then characterized with a vector network analyzer (VNA).

Figure 6a shows the measured photonic Hilbert transform filter RF amplitude frequency response for 12, 16 and 20 taps, respectively. All filters agree well with the theoretical calculations and show very low $(<3 \mathrm{~dB})$ amplitude ripple. All three filters have the same null frequency centred at $16.9 \mathrm{GHz}$, which corresponds to the tap spacing of $\Delta t=1 / f_{c}=59 \mathrm{ps}$. This matches the difference in delay between the comb lines, equal to the micro-ring FSR of $1.6 \mathrm{~nm}$, produced by propagation 

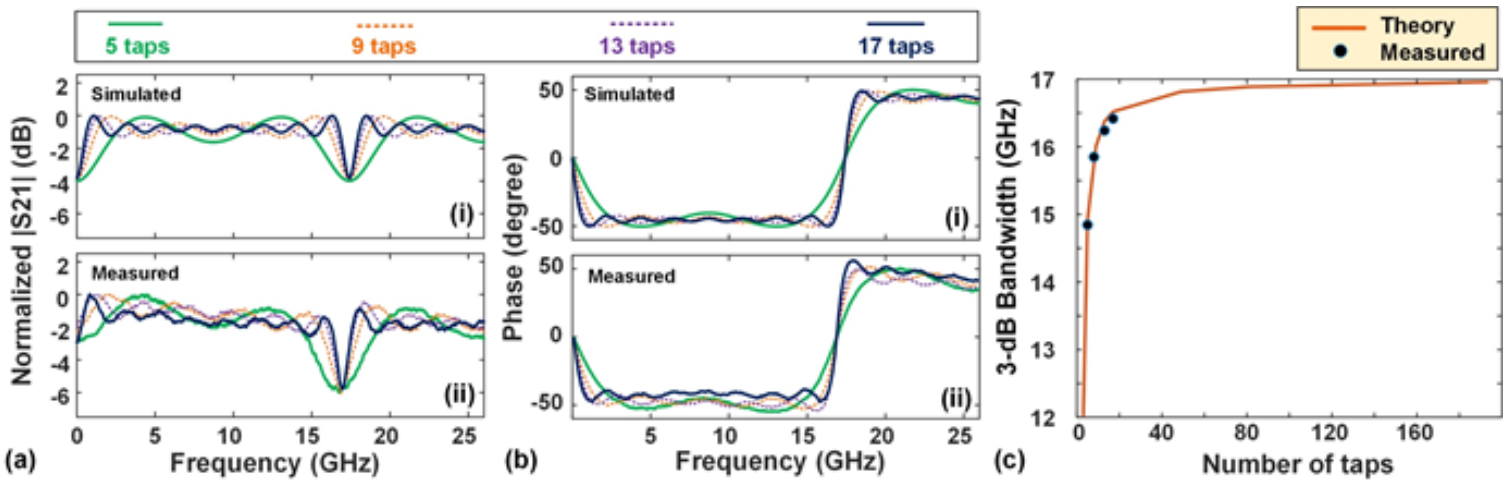

of

(a)

(b)

(c)

the

Fig. 8. (a) and (b) Simulated and measured amplitude and phase response for the FHT for different numbers of taps for a

FHT phase shift of $45^{\circ}$. (c) Simulated and experimental results of 3-dB bandwidth with various taps for a $45^{\circ}$ phase shift.

signal through the $2.122-\mathrm{km}$ long spool of SMF that has the standard dispersion parameter of $D=17.4 \mathrm{ps} / \mathrm{nm} / \mathrm{km}$. The null frequency could be varied by using different fiber lengths to adjust the tap delay spacing or by using tunable dispersion devices. As expected, increasing the number of filter taps increases the filter bandwidth. With a 20-tap filter, the Hilbert transformer exhibited a 3-dB bandwidth extending from $0.3 \mathrm{GHz}$ up to $16.4 \mathrm{GHz}$ - a range of more than 5 octaves - which was a record for any approach to HTs at the time. It is possible to increase this bandwidth even further by using more comb lines in the filter and in fact only a small portion of the generated comb lines was actually used to realize the filter taps. The number of filter taps that could be achieved was in fact limited by the bandwidth of the waveshaper, which was restricted to the C-band, as well as the gain bandwidth of the erbium-doped fiber amplifiers (EDFAs). The amplitude ripple within the passband can be reduced even further by apodizing the tap coefficients compared to the ideal hyperbolic function. Figure 6(b) shows the measured phase response of filters with different numbers of taps, showing a very similar response. Each shows a relatively constant phase of near $-90^{\circ}$ within the passband. There are some deviations from the ideal $-90^{\circ}$ phase at frequencies close to zero and particularly for the null frequency $f_{c}=16.9 \mathrm{GHz}$.

Recently, [126] Tan et. al used a Kerr microcomb having a significantly finer comb spacing of $49 \mathrm{GHz}$ that provided a much larger number of comb lines ( 80 in the $\mathrm{C}$ band) to demonstrate a fractional Hilbert transformer (FHT). By programming and shaping the comb lines according to calculated tap weights, FHTs with phase shifts of $15^{\circ}, 30^{\circ}, 45^{\circ}$, $60^{\circ}, 75^{\circ}$, and $90^{\circ}$ corresponding to fractional orders of $0.17,0.33,0.5,0.67,0.83$, and 1 , respectively, were achieved. A maximum of 17 wavelengths were used, with the space between the 8th and 9th taps and 9th and 10th taps being $0.8 \mathrm{~nm}$, while the remaining spacings were $1.6 \mathrm{~nm}$. This was done in order to maximize the Nyquist zone. The delay line was
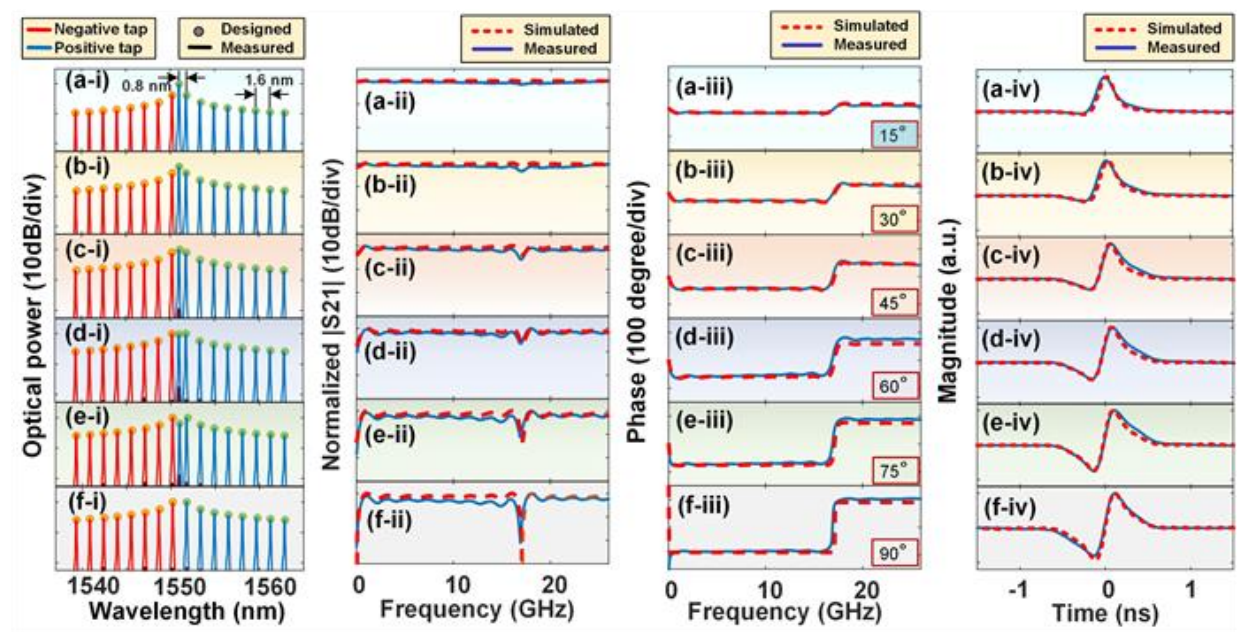

Fig. 9. Simulated (dashed curves) and experimental (solid curves) results of FHT with various phase shifts of (a) $15^{\circ}$, (b) $30^{\circ}$, (c) $45^{\circ}$, (d) $60^{\circ}$, (e) $75^{\circ}$, and (f) $90^{\circ}$. (i) Optical spectra of the shaped microcomb corresponding with positive and negative tap weights (ii) RF amplitude responses with fractional orders of $0.166,0.333,0.5,0.667,0.833$, and 1 . (iii) RF phase responses with phase shifts of $15^{\circ}, 30^{\circ}, 45^{\circ}, 60^{\circ}, 75^{\circ}$ and $90^{\circ}$. (iv) Output temporal intensity waveforms after the FHT. provided by a $2.1-\mathrm{km}$ length of single mode fibre $(\beta=$ $\sim 17.4 \mathrm{ps} / \mathrm{nm} \mathrm{/} \mathrm{km}$ ) that generated a time delay between channels of $\tau=\mathrm{L} \times \beta$ $\times \Delta \lambda=\sim 29.4 \mathrm{ps}$, corresponding to a FSR $_{\mathrm{RF}}$ of $1 / 2 \tau=\sim 17$ GHz. This bandwidth can be increased by using a shorter length of fibre.

The theoretical amplitude and phase response of the FHTs with $15^{\circ}, 30^{\circ}, 45^{\circ}, 60^{\circ}$, $75^{\circ}$, and $90^{\circ}$ phase shifts are shown in Figs. 7 (a)-(f) solid black curves for different numbers of taps. Figures 8(a) and (b) show the RF amplitude and phase 

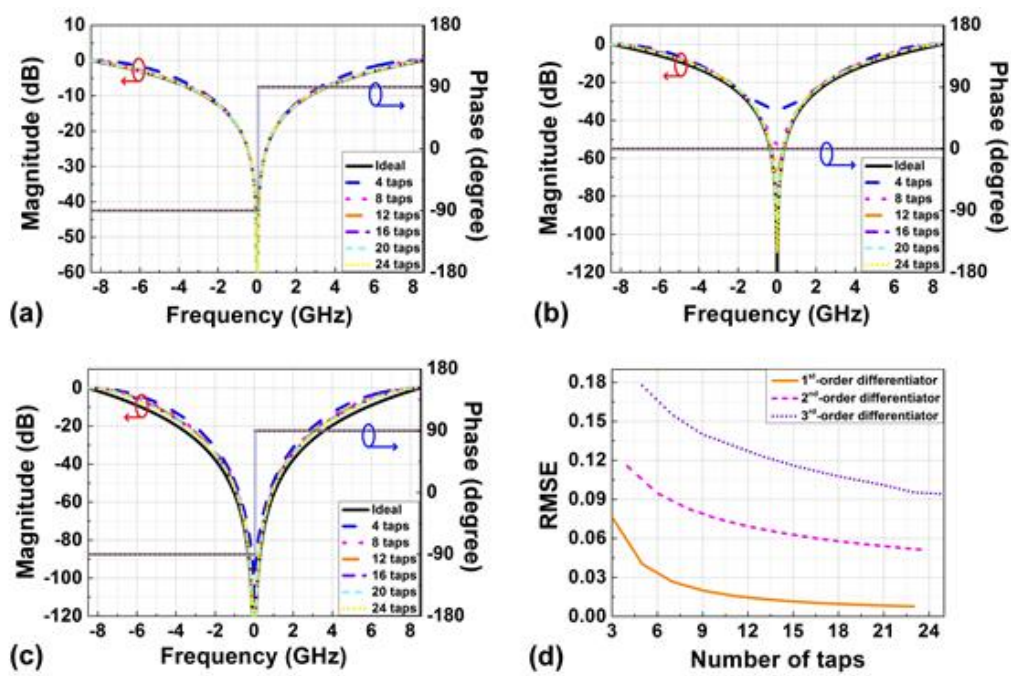

Fig. 10. Simulated RF amplitude and phase responses of the (a) first-, (b) second, and (c) third-order temporal differentiators. (d) RMSEs between calculated and ideal RF amplitude responses of the first-, second-, and third-order intensity differentiators as a function of the number of taps. frequency response for a FHT with a $45^{\circ}$ phase shift with 5, 9, 13 and 17 taps, respectively. Figure $8 \mathrm{c}$ ) shows the $3 \mathrm{~dB}$ bandwidth as a function of the number of taps. As expected, the bandwidth increases with the number of taps. With 17 taps, the operating 3-dB bandwidth for base-band $\mathrm{RF}$ signals was $480 \mathrm{MHz}$ to $16.45 \mathrm{GHz}$ more than 5 octaves - and \pm 0.07 rad phase variation within the $3-\mathrm{dB}$ bandwidth. These numbers represent record performance for FHTs. For a standard $90^{\circ}$ HT the soliton crystal micro-comb provided more than 40 taps, thereby reducing the root-meansquared error (RMSE) and amplitude ripple within the passband [126]. As seen in Fig. 8 (c), the theoretical $3-\mathrm{dB}$ bandwidth increases rapidly with the number of taps up to 17 taps, after which it levels off, so that 17 to 20 taps is close to the optimum number.

Figure 9 (ii)-(iii) presents the simulated (red dashed curves) and measured (blue solid lines) response of the fractional Hilbert transformers with variable orders ranging from $0.166-0.833$ (phase shifts $15^{\circ}-90^{\circ}$ ). The normalized frequency response for both the magnitude and phase of the FHTs are shown together with the temporal response (Fig. (iv)). The amplitude variations are less than $+/-1.5 \mathrm{~dB}$ between $480 \mathrm{MHz}$ and $16.45 \mathrm{GHz}$ and the phase variations are about $\pm 0.07 \mathrm{rad}$ within the 3-dB passband. System demonstrations of a real-time FHTs applied to Gaussian input pulse signals were also performed. Fig. 9 (iv) shows a pulsed waveform after processing by the fractional Hilbert transformer along with the simulated results (Fig. 9 (iv), red dashed curves). The detailed performance parameters are listed in Table I of Ref. [126]. As can be seen, the measured curves closely match the theoretical counterparts, achieving good performance that agrees well with theory.

In comparing the earlier results achieved with the 200GHz FSR microcomb with the more recent FHT results achieved


Fig. 11. (a) Optical spectrum of the generated Kerr comb in a 300-nm wavelength range. Inset shows a zoom-in spectrum with a span of $\sim 32$ nm. (b)-(d) Measured optical spectra (red solid) of the shaped optical combs and ideal tap weights (green crossing) for the first-, second-, and third-order intensity differentiators.
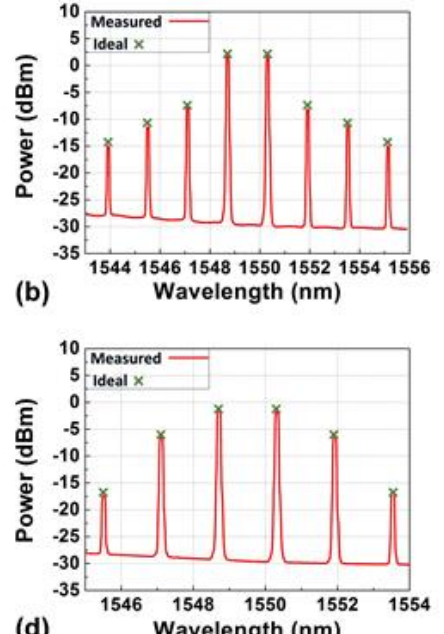
with the soliton crystals having a 49-GHz FSR spacing, it is clear that the soliton crystal based results yield superior performance over the 200$\mathrm{GHz}$ comb-based transformer in terms of bandwidth, mainly because of the larger number of available taps. Nonetheless, we note that it is not always the case that a smaller FSR comb is necessarily better. The $200-\mathrm{GHz}$ devices are capable of reaching extremely high $\mathrm{RF}$ bandwidths, particularly in tunability, approaching $100 \mathrm{GHz}$, since this represents the Nyquist zone of these combs, whereas the $49 \mathrm{GHz}$ devices are limited to about $25 \mathrm{GHz}$. In summary, our good agreement with theory confirms that the approach of using microcombs is a very effective way to implement high-speed reconfigurable HTs and FHTs with lower complexity, reduced footprint and cost.

\section{DIFFERENTIATION}

The fundamental operation of differentiation is 

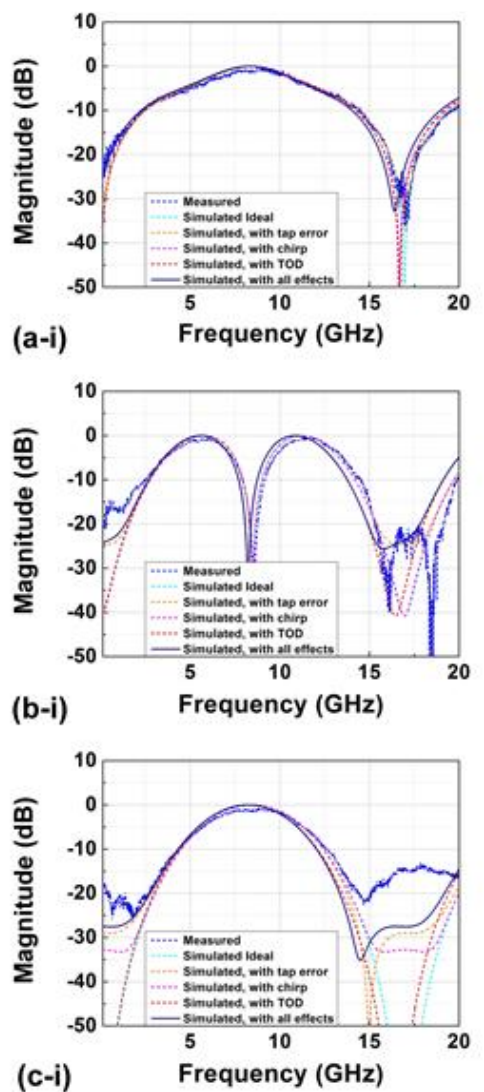

(c-i) Frequency (GHz)

Fig. 12. Measured and simulated RF amplitude and phase responses of (a-i)-(a-ii) the first-order, (b-i)-(b-ii) second-order, and (c-i)-(c-ii) third-order intensity differentiators.
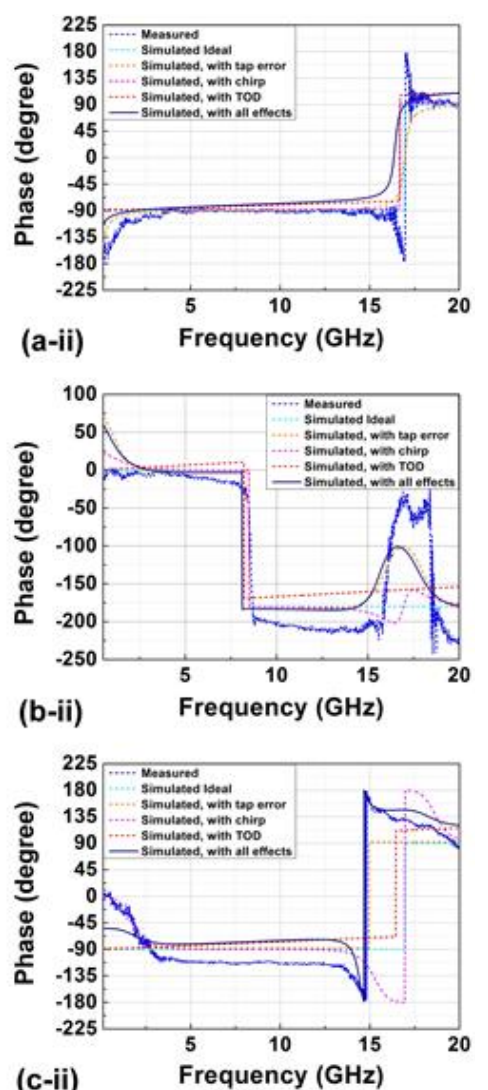

(c-ii)

a key function in RF and microwave signal processing. It has wide applications for RF spectral analysis, ultra-wideband (UWB) generation, and RF spectral filters [167171]. Significant progress has been made on RF integral differentiators via photonicbased technologies [172-184] using selfphase modulation (SPM) [172,173] and cross-phase modulation (XPM) [177-179] methods, frequency discriminators and cross-gain modulation in semiconductor optical amplifiers (SOA) [176]. Compared to conventional integral differentiation, fractional differentiation has many more unique and powerful applications $[127,182,183]$. It has played an important role in many different fields including electricity and electronics, mechanics, chemistry, biology, and economics. Perhaps its most important applications have been in image edge detection, control theory, and mechatronics [182,183]. Interestingly though, despite these wide benefits and applications, photonic based fractional differentiators have received little attention compared to other functions.

For the most part, photonic differentiators - both integral and fractional - have focused on generating the derivative of the complex optical field, rather than the actual RF signal. For example, a photonic differentiator based on a dual-drive MZM together with an RF delay line, [174] although successful, was limited in processing speed by the bandwidth of the RF delay line. Photonic differentiators based on optical filters [175] feature high speeds of up to 40-Gb/s, although they only work for a fixed (and typically integral) differentiation order and generally lack flexibility or reconfigurability. To implement highly reconfigurable differentiators, transversal schemes have been investigated using discrete laser arrays [169, 180, 181]. While this is a powerful and attractive approach, it also presents some limitations, chiefly in the number of wavelengths that can be supplied. Increasing the number of lasers to provide more taps dramatically increases the system size, cost, and complexity, thus limiting the processing performance. Using a single source that can simultaneously generate a large number of high-quality wavelength channels would be highly advantageous. Recently [109], a photonic RF integral differentiator with 8 taps, achieved using a 200-GHz FSR micro-comb source was demonstrated, that generated the $1^{\text {st }}, 2^{\text {nd }}$, and $3^{\text {rd }}$ order derivatives operating at RF bandwidths up to $17 \mathrm{GHz}$ with a potential bandwidth of $100 \mathrm{GHz}$.

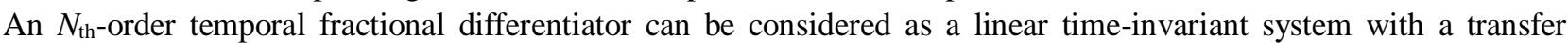
function given by [168]

$$
H_{N}(\omega) \propto(j \omega)^{N}
$$

where $j=\sqrt{-1}, \omega$ is the RF angular frequency, and $N$ is the differentiation order which can be fractional and even complex $[184,185]$. According to Equation 5, the amplitude response of a temporal differentiator is proportional to $|\omega|^{N}$, whereas the phase response has a linear profile, with a phase jump of $N \pi$ at null frequencies.

Using the transverse filter approach to RF photonic differentiators yields a finite set of delayed and weighted replicas of the input RF signal in the optical domain, which is then combined upon detection. The transfer function from Eq. (5) becomes

$$
H_{N}(\omega)=\sum_{n=0}^{M-1} a_{n} e^{-j \omega n T}
$$




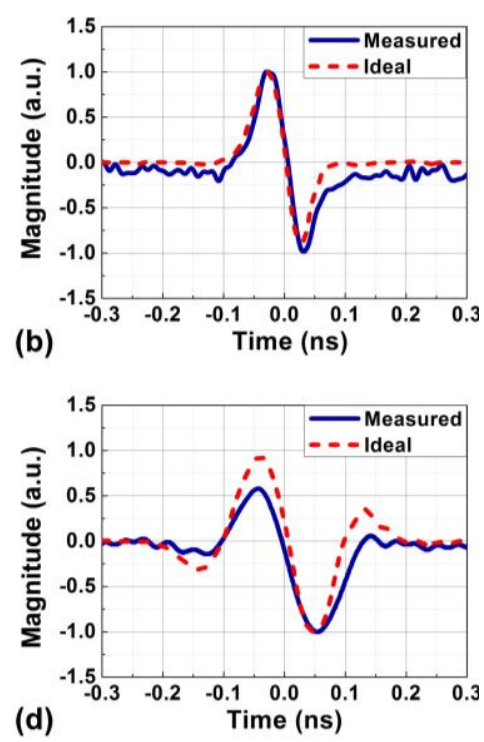

(d)

where $M$ is the number of taps, $a_{n}$ is the tap coefficient of the $n$-th tap, and $T$ is the time delay between adjacent taps. It should be noted that differentiators that achieve the function based on Eq. (6) are intensity RF differentiators, i.e., the output RF signal after being combined upon detection yields an exact differentiation of the input RF signal, in contrast to optical field differentiators that produce the derivative of complex optical fields [167,171,153,154,185-187].

Recently, [109] the first use of a micro-comb to achieve RF differentiation was reported, demonstrating integral $1^{\text {st }}$, $2^{\text {nd }}$, and $3^{\text {rd }}$ order intensity differentiators. That demonstration was achieved with a 200GHz FSR Kerr micro-comb using 8, 6, and 6 taps, respectively. It achieved a dynamic range, determined by the EDFA, of $\sim 30 \mathrm{~dB}$. More taps are needed when the differentiation order increases, and for a

fixed number of taps, increasing the order of differentiation also increases the required power dynamic range. In order to obtain better performance for a given number of taps, the operation bandwidth of the integral differentiators was decreased to half of the Nyquist frequency. To implement the temporal differentiator in Eq. (5), tap coefficients in Eq. (6) were calculated based on the Remez algorithm [188]. The calculated tap coefficients for $1^{\text {st }}, 2^{\text {nd }}$, and $3^{\text {rd }}$ order differentiation are listed in Table I of [109]. As for demonstrations of other RF functions, since the $200 \mathrm{GHz}$ Kerr comb was not flat, real-

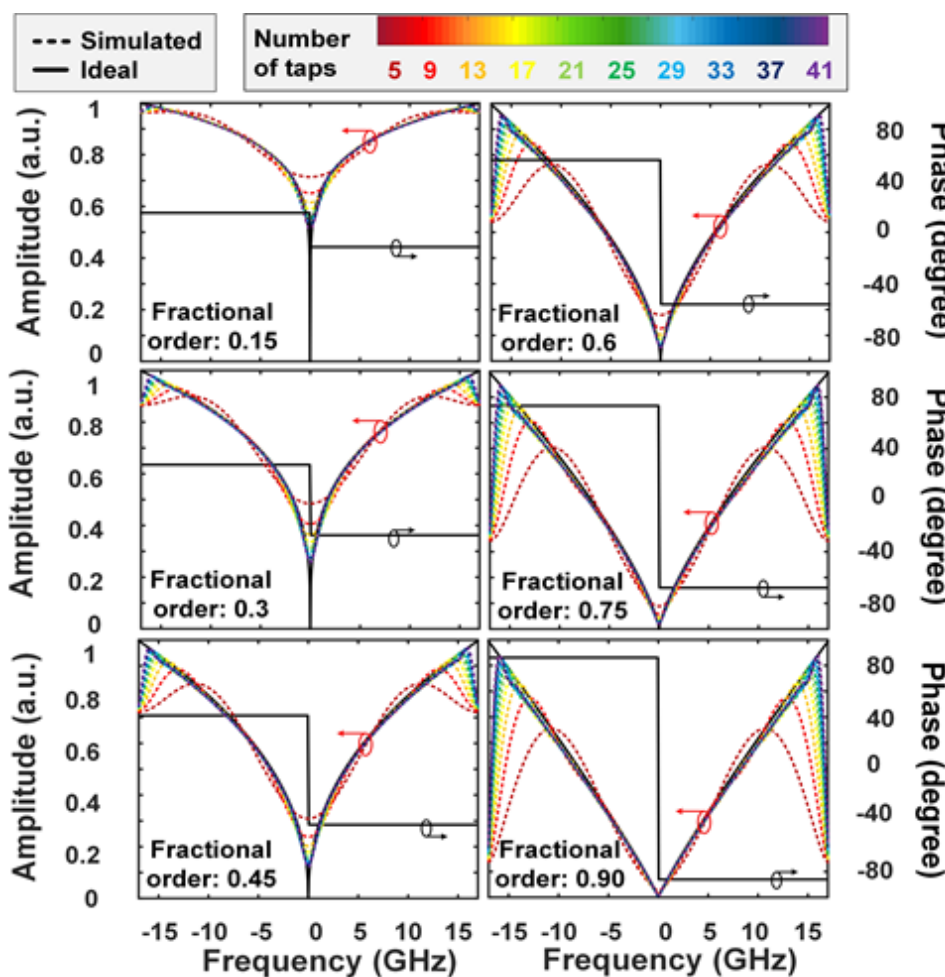
time feedback control was used to increase the accuracy of the comb shaping. Both the phase and amplitude responses of the $1^{\text {st }}, 2^{\text {nd }}$, and $3^{\text {rd }}$ order differentiators are shown in Figs. 10 (a)-(c) for different numbers of taps. As the number of taps increases, the discrepancies between the ideal and realized amplitude responses of the transversal filter based differentiators are improved for all three orders. In contrast the phase response of the transversal filters is identical to that of the ideal differentiators regardless of the number of taps.

The shaped optical comb spectra shown in Figs. 11 (b)-(d) reveal a good match between the measured comb lines' power (red solid line) and the calculated ideal tap weights (green crosses). Figures 12 (a-i), (b-i), and (c-i) show the measured and calculated amplitude response of the differentiators. The corresponding phase response is depicted in Figs. 12 (a-ii), (b-ii), and (c-ii) where it is clear that all three differentiators agree well with theory. The FSR of the RF response spectra was $\sim 16.9 \mathrm{GHz}$, consistent with expectations given the time delay between

Fig. 14. Simulated transfer function of different fractional differentiation orders with varying number of taps. 

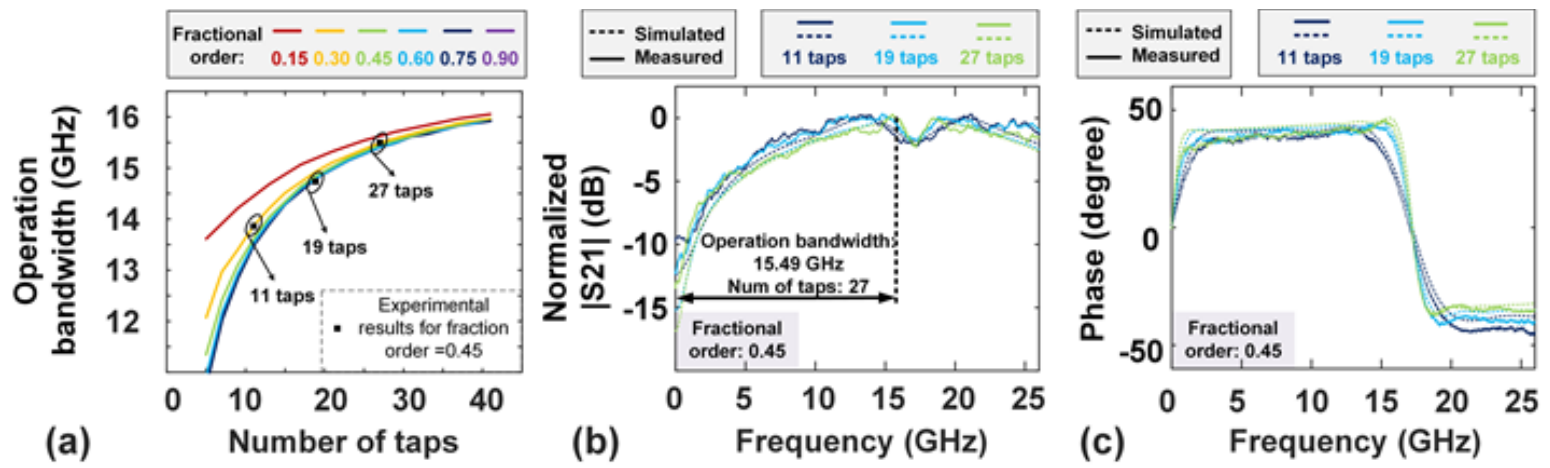

Fig. 15. (a) Relationship between the number of taps and operation bandwidth. (b, c) Experimentally demonstrated fractional differentiator with varying number of taps.

adjacent taps. By adjusting the FSR of the transversal filter through varying the dispersive fibre delay or programming the tap coefficients, a variable operational bandwidth for the intensity differentiator can be achieved, which is advantageous for the changing requirements of different applications. In particular, the ability to change the order of the differentiator simply by changing the tap weights offers a powerful way of dynamically changing the functionality of the system in real time without needing to modify any hardware. This is a feature that is not common to other approaches to differentiation, particularly optical filter-based systems.

Systems demonstrations of real-time signal differentiation were also performed for Gaussian input pulses having a fullwidth at half maximum (FWHM) of $\sim 120$ ps, as shown in Fig. 13 (a). The generated waveforms of the output signals, representing the different order derivatives, are shown in Figs. 13 (b)-(d) (blue solid curves), together with the theoretical results (red dashed curves). The experimental Gaussian pulse shown in Fig. 13 (a) was used as the input RF signal for the theoretical calculations. The measured curves closely match theory. Unlike the optical field differentiators $[167,171,153,154,185-187]$, the temporal derivatives of the RF intensity profiles can be observed, indicating that intensity differentiation was successfully achieved. For the $1^{\text {st }}, 2^{\text {nd }}$, and $3^{\text {rd }}$ order differentiators, the calculated RMSE between the


Fig. 16. Optical spectra of the shaped micro-comb for different fractional orders. measured and theoretical curves are $\sim 4.15 \%, \sim 6.38 \%$, and $\sim 7.24 \%$, respectively.

Recently, [127] Tan et.al reported the first fractionalorder photonic RF differentiator that operates directly on the RF signal rather than the complex optical field. It was based on a record-low 49-GHz-FSR Kerr micro-comb source, which provided a large number of comb lines $(80$ in the $\mathrm{C}$ band), resulting in a broad operation bandwidth of 15.49 GHz. By programming and shaping the comb lines according to the calculated tap weights, reconfigurable arbitrary fractional orders ranging from 0.15 to 0.9 were demonstrated. System demonstrations of real-time fractional differentiation of Gaussian input pulses were also performed. The good agreement between theory and experiment confirmed that this approach as an effective way to implement high-speed reconfigurable fractional differentiators with reduced footprint, lower complexity, and potentially lower cost. 


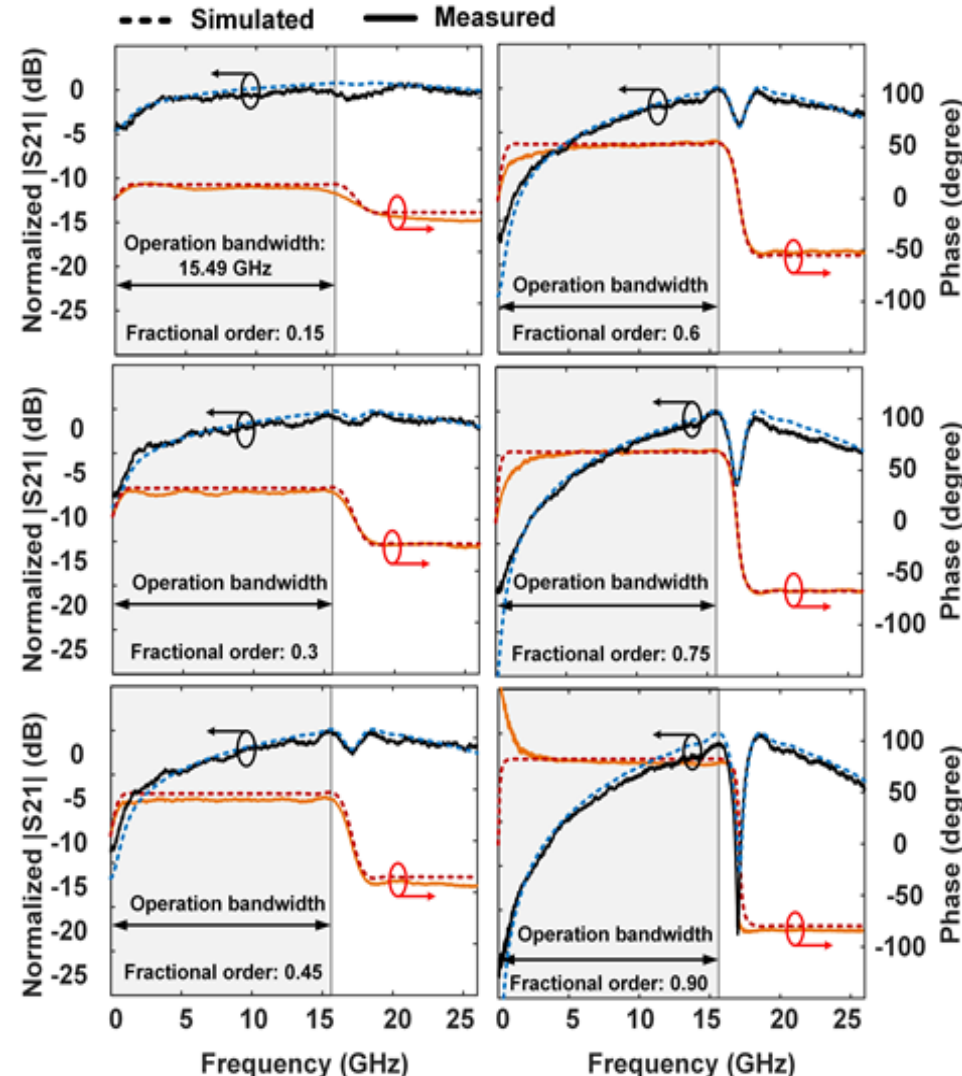

Fig. 17. Simulated and measured the transmission response of the fractional differentiator at different orders ranging from 0.15 to 0.90 .
The success of the differentiator resulted from both the use of soliton crystals combined with the low FSR of $48.9 \mathrm{GHz}$, that generated over 80 wavelengths, or taps, in the C-band. The performance of the system was greatly aided by the soliton crystal's extremely robust and stable operation, ease of initiation and generation and as well as its much higher intrinsic efficiency, all of which are enabled by the integrated CMOS-compatible platform. The relationship between the number of taps and the performance of the fractional differentiator was also investigated. Figure 14 shows the theoretical transfer function of the fractional order differentiator for six different orders and for varying numbers of taps, compared to the ideal theoretical results. As can be seen, the operational RF bandwidth of the differentiator - within which the slope coefficients of the simulated and ideal amplitude responses match closely-increases with the number of taps. Figure 15 (a) shows that the number of taps is critical to the operational bandwidth for fractional differentiators, which is evident in the experimental results for the fractional order of 0.45 (Figs. 15 (b) and (c)). When the number of taps was increased to 27 , the bandwidth of the fractional differentiator reached 15.49 $\mathrm{GHz}$, equivalent to over $91 \%$ of the Nyquist band. In the experiments, up to 29 comb lines were employed to generate the taps. To increase the number of taps, the optical signal-to-noise ratio, which was subject to the optical amplifiers' noise, would need to be increased.

The shaped comb spectra used to generate the derivatives are shown in Fig. 16, where it is evident that the optical power for each comb line matched the designed tap coefficients well.. The transmission frequency response of the fractional differentiator was characterized with a VNA. The power response of the differentiator showed a bandwidth ranging from DC to $15.49 \mathrm{GHz}$. As shown in Fig. 17, the range of fractional orders that the system was able to achieve was a result of the close agreement between theory and experimental in terms of the slope coefficients of the power response and phase shift in the phase response. Figure 18 shows the time domain performance of the fractional differentiator for all orders, using a baseband RF Gaussian pulse with a FWHM of 200ps (Fig. 18) as the input signal,
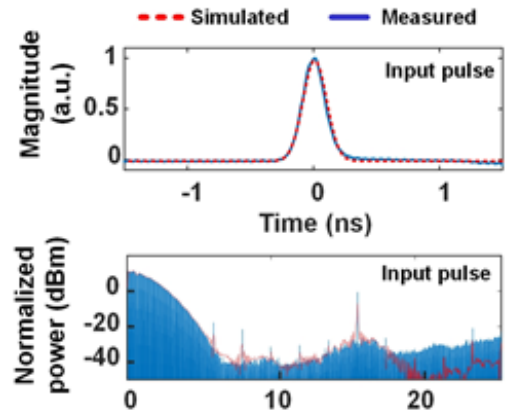

(a)

Frequency $(\mathrm{GHz})$
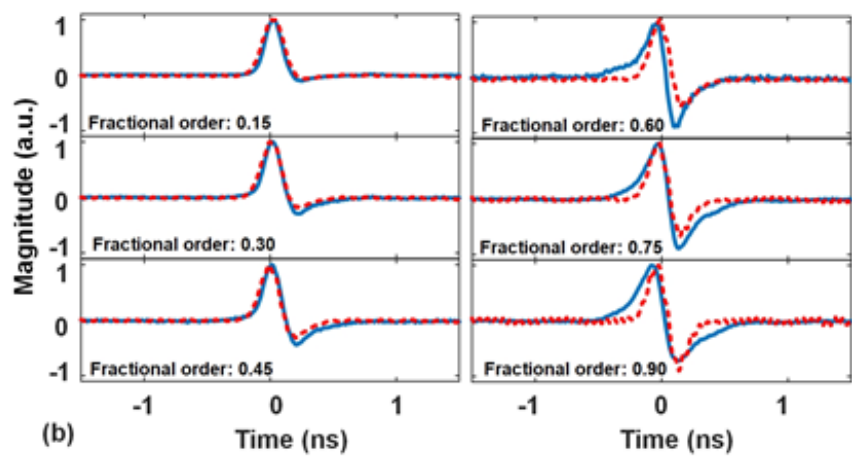

Fig. 18. Simulated and measured RF Gaussian pulse output temporal intensity waveform after the fractional differentiator. 
generated by an arbitrary waveform generator. The good agreement with theory reflects the range of orders that are achievable with our approach to realizing a reconfigurable fractional differentiator.

\section{INTEGRATION}

Temporal integration is another key fundamental function in signal processing systems. Photonic techniques offer a broad bandwidth, in contrast to electrical integrators that are subject to the electronic bandwidth bottleneck, along with a strong immunity to EMI, as well as low loss [79,80,189]. Significant work has been reported on photonic integrators, including gratings based approaches [190-192] and methods using micro-ring resonators (MRRs) [15,42,193]. These approaches have achieved optical integration with a time resolution as short as 8 ps [193] as well as offering a large timebandwidth product due to the availability of very high-Q resonant structures. However, these approaches still face limitations for RF signal processing, including the fact that they are not adjustable in their performance parameters such as temporal resolution or length of integration window. Hence, they lack the flexibility to process RF signals with different bandwidths and integration time windows. Probably more importantly, those approaches rely on processing the optical signals - they cannot directly process RF signals without electrical to optical interfaces. Another important approach to photonic integrators is based on transversal structures, that offers a high degree of reconfigurability and accuracy owing to the parallel scheme where each path can be controlled independently [194-196]. By tailoring the progressive delay, RF signal integration with a reconfigurable operational bandwidth can be achieved [194-196]. Still, however, these still face limitations arising from the limited number of channels in cases where diode laser arrays or electro-optical comb sources were employed. These approaches have a significant trade-off between the number of wavelengths and system complexity and ultimately they result in a limited number of channels which, and in turn, limits the time-bandwidth product.

Integrated Kerr micro-combs [38, 47, 68-70,118,197] offer many advantages for RF integration, including a very high higher number of wavelengths as well as a greatly reduced footprint and complexity. Recently, a highly reconfigurable



Fig. 19. Schematic diagram of the photonic RF integration. photonic RF temporal integrator was demonstrated with an integrated soliton crystal micro-comb source [123], where the RF signal was imprinted onto the micro-comb lines and progressively delayed via dispersion, and then summed upon detection. The large number of taps, or wavelengths (81) used in those experiments resulted in a large integration time window of $\sim 6.8 \mathrm{~ns}$ with a time resolution as short as $\sim 84 \mathrm{ps}$. The integrator was successfully tested with different input signals, with experimental results matching well with theory.

Figure 19 shows the operation principle of the transversal filter based photonic RF integrator. The integration process can be achieved via a discrete time-spectrum convolution operation between an RF input signal $f(t)$ and a flattened micro-comb. With a delay step $\Delta t$, the operation is:

$$
y(t)=\sum_{k=1}^{N} f(t+k \cdot \Delta t)
$$

where $j=\sqrt{-1}, N$ is the total number of wavelengths. After the replicas of $f(t)$ are progressively delayed and then summed, the temporal integration of the function $f(t)$ results, with a time resolution given by [194] $\Delta t$, an operation bandwidth of $1 / \Delta t$, and a total integration time window of $T=N \times \Delta t$. The frequency-domain transmission response of an ideal continuous, or non-discrete, integrator is linear to $1 /(j \omega)$, where $\omega$ is the angular frequency. The frequency transmission response of the discrete integrator is a low pass sinc filter [111,117], given by

$$
H(\omega)=\sum_{n=0}^{N-1} e^{-j \omega n \Delta t}
$$


The experimental setup of the photonic RF integrator was similar to that shown in Figure 4, and was based on a soliton crystal micro-comb with an FSR of $\sim 49 \mathrm{GHz}$. The soliton crystal micro-comb featured very low intensity noise to the extent that it has even been used as a photonic local oscillator [120]. Thus, the noise of the micro-comb source did not introduce any observable deterioration in the overall noise performance of the integrator. After flattening the micro-comb spectral lines with a WaveShaper, the RF signal was imprinted onto the comb lines via an electro-optical modulator (EOM). The replicas were progressively delayed by a $\sim 13 \mathrm{~km}$ spool of standard optical fibre SMF and summed upon photodetection with a high-speed photodetector. The delay between the adjacent wavelengths $(\Delta t)$ was set by the dispersion, length of fibre and spacing between the comb lines, and this in turn determined the resolution of the integrator [194]. The temporal resolution was $\sim 84 \mathrm{ps}$ and in theory can be made arbitrarily small by reducing the amount of dispersion in the delay line, although with a trade-off that the integration window also decreases. Sixty comb lines in the C-band were selected, yielding an integration time window $(T=$ $N \times \Delta t$ ) of $60 \times 84 \mathrm{ps}=5.04 \mathrm{~ns}$ (yellow shaded region in Fig. 20). The operation bandwidth of the integrator was $1 / \Delta t$
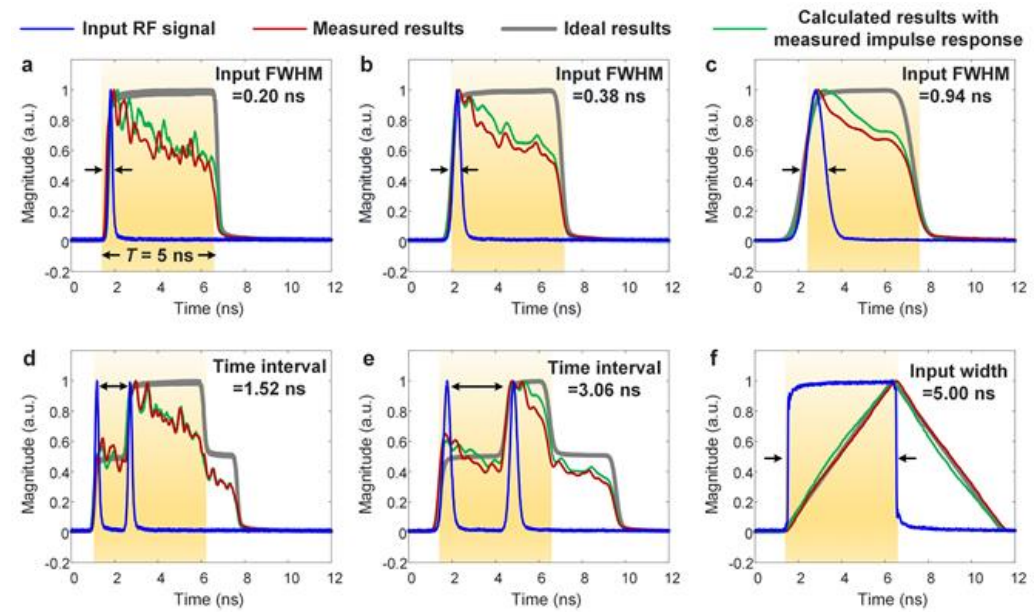

Fig. 20. Experimental results of the micro-comb-based RF integrator after comb optical power shaping for input (a-c) Gaussian pulses with FWHM of 0.20, 0.38 and $0.94 \mathrm{~ns}$, (d-e) dual Gaussian pulses with time intervals of 1.52 and $30.6 \mathrm{~ns}$, and (f) a triangular waveform with a width of $5.00 \mathrm{~ns}$. The blue curves denote the input signal, the red curves denote the measured integration results, the gray curves denote the ideal integration results, and the green curves denote the integration results calculated with the measured impulse response of the system.

$=11.9 \mathrm{GHz}$. Signal integration for

different RF input signals was performed. The red curves in Figs. 20(a)-(c) show the results for Gaussian pulses (blue curves) with a FWHM from $0.20 \mathrm{~ns}$ to $0.94 \mathrm{~ns}$, with the integration window $T(\sim 5 \mathrm{~ns})$ matching with theory well (5.04 ns). Figures $20(\mathrm{~d})-(\mathrm{e})$ show the results for dual Gaussian pulses with time intervals of $1.52 \mathrm{~ns}$ and $3.06 \mathrm{~ns}$, where it is clear that the experimental results (red curves) illustrate the performance of the integrator by exhibiting three distinct intensity steps in the integration waveforms. The left step corresponds to the integration of the first pulse while the middle step reflects the integration of both of the two initial pulses, and finally the right step shows the integration of only the second pulse since it is beyond the integration window of the first pulse. The integrator was further tested with a rectangular input waveform having an equal width to the integration window $(5 \mathrm{~ns})$. The measured integrated waveform exhibited a triangular shape that matched well with theory (gray curve).

Figure 20 shows small residual discrepancies between experiment (red curves) and theory (grey curves). The errors were a result of the non-ideal impulse response of the system, caused by a number of relatively small effects that include gain variation with wavelength of the optical amplifier, modulator and photodetector. To verify this, the impulse response of the system was measured with a Gaussian pulse input. Since the time resolution of the system $(\sim 84 \mathrm{ps})$ was much less than the pulse width, the wavelength channels were separated into multiple subsets (each with a much larger spacing between the adjacent comb lines and thus obtained a temporal resolution larger than the input pulse duration), and their impulse responses measured sequentially. Figure 21(a) shows the measured impulse response of the system, which was not flat
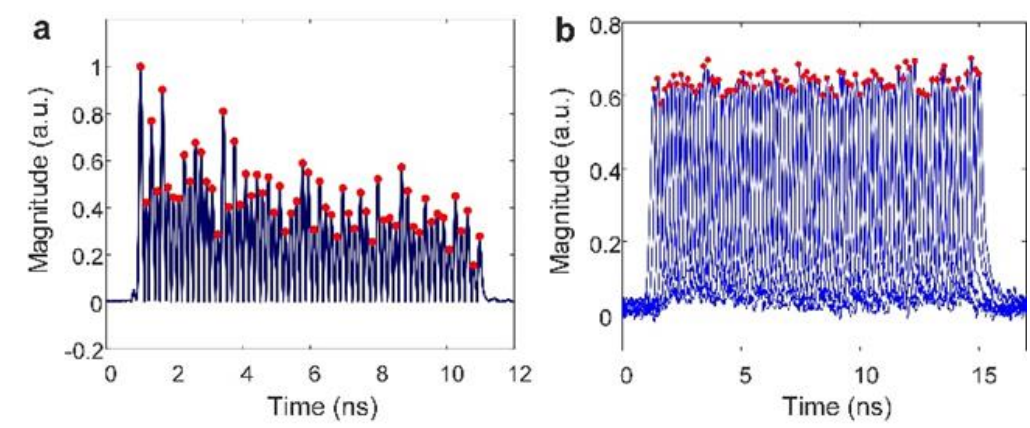

Fig. 21. Measured impulse response of the integrator (a) after comb optical power shaping and (b) after impulse response shaping using a Gaussian RF input pulse. 
even when the comb lines were perfectly flattened. The measured impulse response and the input RF signal in Fig. 20 was used to calculate the corresponding integral output, with the results matching the experiment well, showing that the errors were induced by the non-ideal impulse response of the system.

These errors are in fact straightforward to compensate for. To reduce the errors, a more accurate comb shaping was used where the error signal of the feedback loop was generated directly by the measured impulse response, instead of the optical power of the comb lines. Thus this implicitly had the wavelength dependence of the components built-in. A flattened
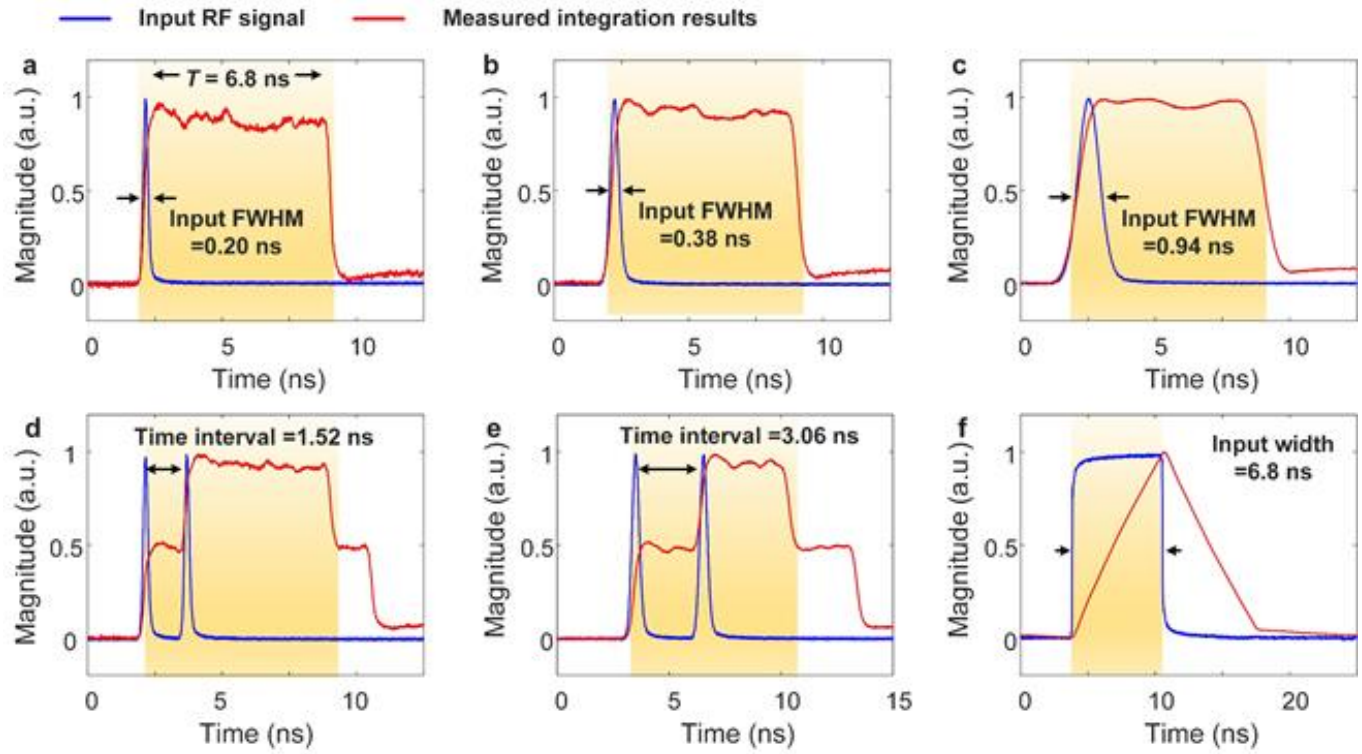

Fig. 22. Experimental results of the micro-comb-based RF integrator after impulse response shaping for input (a-c) Gaussian pulses with FWHM of $0.20,0.38$ and $0.94 \mathrm{~ns}$, (d-e) dual Gaussian pulses with time intervals of 1.52 and 3.06 $\mathrm{ns}$, and (f) a triangular waveform with a width of $5.00 \mathrm{~ns}$. The blue curves denote the input signal, the red curves denote the measured integration results.

impulse response was obtained (Fig. 21(b)), which was much closer to the ideal impulse response than Fig. 21(a). The integration was then performed with the same RF inputs, the results of which are shown in Fig. 22. Note that during this measurement, 81 wavelength channels were enabled by the impulse response shaping process, so that the integration time window $(T=N \times \Delta t)$ increased to $81 \times 84 \mathrm{ps}=6.804 \mathrm{~ns}$, resulting in an operation bandwidth of $1 / 84 \mathrm{ps}=11.9 \mathrm{GHz}$ and a time-bandwidth product of $6.804 \mathrm{~ns} \times 11.9 \mathrm{GHz}=\sim 81$ (approximately equal to the number of channels $N$ ). The measured integrals (red curves, Fig. 22) show significantly better agreement with theory, indicating the success of the compensating impulse response shaping method as well as the overall power and feasibility of this approach to photonic RF integration.

\section{DISCUSSION}

We have reviewed recent progress on the use of Kerr micro-combs for high bandwidth temporal RF and microwave signal processing, focusing on integral and fractional order Hilbert transformers, high order and fractional differentiators, both with continuously tunability in their order, and finally RF integrators. Micro-combs produce a large number of comb lines that greatly increase the performance and processing bandwidth of RF systems in terms of both the amplitude and phase response. They have shown their powerful capabilities for RF signal processing due to their large number of comb lines, their compact footprint, and large comb spacings that can be generated compared to all other approaches such as those using electro-optic combs. The devices reached operation bandwidths of $\sim 16 \mathrm{GHz}$, for the $49 \mathrm{GHz}$ devices and $\sim 25$ $\mathrm{GHz}$ for $200 \mathrm{GHz}$ FSR combs, evaluated in the frequency domain using Vector Network Analyzers and in the time domain with Gaussian pulse input signals.

For transversal frequency-domain signal processors such as Hilbert transformers and differentiators, the number of wavelengths determines the number of taps, which in turn directly determines the system performance. We have highlighted the advantages of using Kerr micro-combs as the basis for RF photonic microwave transversal filters, illustrating the tradeoffs between using widely spaced micro-combs $(200 \mathrm{GHz})$ and record-low spaced micro-combs (49 
$\mathrm{GHz}$ ) in terms of performance. The greater number of lines supplied by the $49 \mathrm{GHz}$ comb (80 versus 20 for the $200-\mathrm{GHz}$ device) yielded significantly better system performance. On the other hand, the 49-GHz device is more limited in operational bandwidth, being restricted to roughly the Nyquist zone of $25 \mathrm{GHz}$, whereas the 200-GHz devices were able to reach RF frequencies well beyond what conventional electronic microwave technology can achieve.

Two types of combs employed to realize RF functions were qualitatively very different. Apart from the spacings being very different, the widely spaced $200 \mathrm{GHz}$ FSR microcomb operated in a quasi-coherent state that did not feature solitons, whereas the low spaced microcomb with an FSR of $49 \mathrm{GHz}$ operated in a state referred to as soliton crystals. Within the optical $\mathrm{C}$ band, the $49 \mathrm{GHz}$ spaced microcomb enabled up to 90 comb lines, yielding significantly better performance for the temporal signal processors than the $200 \mathrm{GHz}$ spaced microcomb which only allowed 20 taps in the C-band. Further, the soliton crystal states offered many other advantages such as much lower noise, higher stability, and much easier generation including the ability to even initiate soliton crystals through simple manual tuning of the pump wavelength. However, on the other hand the $49 \mathrm{GHz}$ combs generally yielded much smaller Nyquist bandwidths typically near $\sim 16 \mathrm{GHz}$. For the transversal temporal signal processors such as the integrators, the large number of wavelengths brought about a large number of broadcasted RF replicas, thus yielding a large integration window for the integrator. The temporal response of the integrator was measured with a diverse range of RF inputs, verifying a integration window of $6.8 \mathrm{~ns}$ and a time feature as fast as 84 ps. The good agreement with theory and experiment verified that this approach is an effective way to implement high-speed reconfigurable signal processing featuring high processing bandwidths, for future ultra-high-speed microwave systems.

We believe that micro-combs will bring further benefits to RF photonic signal processing in many respects. First, the coherent nature of the soliton states will enable more advanced RF functions such as wideband frequency conversion and clock generation. Maturing nanofabrication techniques have allowed the realization of high-Q MRRs for comb generation with FSRs ranging from 10s' of GHz [119] up to the THz regime [47], covering the full RF bands of interest for almost any integrated RF system. The wide available FSR spacing of optical micro-combs allows for a large Nyquist zone up to 100 's of GHz. This is well beyond the processing bandwidth of electronic devices and is also challenging for mode-locked lasers and OFCs obtained by EO modulation. With tailored dispersion, ultra-wideband bandwidth micro-combs - even up to octave-spanning and beyond, [71] can be achieved, thus enabling a very large number of wavelength channels with a single on-chip source, to work in conjunction with broadband opto-electronic equipment. This approach enables significantly enhanced wavelength-division parallelism for massive data transmission and processing [62-64] for many applications including radio-over-fibre systems, for example.

Perhaps most importantly, the high reconfigurability of micro-comb based programmable RF photonic transversal filters enables one to achieve highly versatile processing functions with dynamic real-time reconfigurability so that the same system can be dynamically reprogrammed to perform a wide range of different functions without having to alter any hardware. By simply programming and shaping the comb lines according to different specific tap coefficients, the same physical equipment can be employed to achieve multiple signal processing functions. Such a high degree of reconfigurability is unprecedented for photonic RF signal processors and enables highly reconfigurable processing functions and high processing accuracy that typically cannot be obtained by passive photonic integrated circuits or via optical analogue signal processing. Further, very wide operational RF bandwidths can be achieved for diverse computing requirements of many practical applications. Photonic transversal filters are essentially equivalent to filters achieved via purely digital signal processing, but implemented by photonic hardware.

There are a number of factors that can lead to tap errors during the comb shaping, thus leading to non-ideal impulse responses of the system as well as deviations between the experimental results and theory. These factors mainly include the instability of the optical micro-combs, the accuracy of the waveshapers, the gain variation with wavelength of the optical amplifier, the chirp induced by the optical modulator, and the third-order dispersion of the dispersive fibre. To combat this, real-time feedback control paths can be employed to effectively reduce the errors. In this approach, the power of the comb lines is first detected by an optical spectrum analyser (OSA) and then compared with the ideal tap weights, generating an error signal that is fed back into the waveshaper to calibrate the system and achieve accurate comb shaping. To further reduce the tap errors and improve the accuracy of comb shaping, the error signal of the feedback loop can be generated directly by the measured impulse response, instead of the raw optical power of the comb lines. In this approach, replicas of an RF Gaussian pulse are measured at all wavelengths to obtain the impulse response of the system, whose peak intensities are further extracted to obtain accurate RF-to-RF wavelength channel weights. Following this, the extracted channel weights are subtracted from the desired weights to obtain an error signal that is used to program the loss of the Waveshaper. After several iterations of the comb shaping loop, an accurate impulse response that compensates the nonideal impulse response of the system can be obtained, thus significantly improving accuracy of the RF photonic transversal 
filter based signal processors. This only requires a one-time calibration process.

Future challenges for microcomb based RF systems centre around improvements to the micro-combs themselves as well as the overall RF systems with which they are embedded. For the microcombs, one important point is that their intrinsic quality and performance - ie., coherence, linewidth, stability, etc. -is already at a state that is beyond the requirements for most microwave applications. Microcombs have been the basis of extremely demanding demonstrations that have yielded record performance in many different applications. These include optical frequency synthesizers [68], ultrahigh bandwidth optical data communications [62, 64], and high-performance X-, K-, and U-band microwave signal generation [118-120] achieving phase noise as low as $-110 \mathrm{dBc} / \mathrm{Hz}$ (at $10 \mathrm{kHz}$ offset) [119].

The requirements for RF transversal filter applications, on the other hand, are much more modest. A full treatment of this issue is beyond the scope of this work and we refer the reader to other more in-depth and broader reviews of microcombs [47, 69-72]. Nonetheless, there are still many challenges that ultimately need to be addressed. In particular, aside from achieving ever smaller FSRs discussed above, there is the ongoing need to increase the microcomb efficiency (conversion of pump energy to comb lines) as well as stability, robustness, repeatability in generation, and the degree of integration.

Soliton crystals represent a significant breakthrough in improving the efficiency and robustness of the combs. This is reflected in the demonstration mentioned above on ultrahigh bandwidth optical data communications [64]. DKS states achieved a world record in 2017 for high bandwidth data transmission from a single source chip - a result of their very high coherence, fidelity and stability [62]. That record was superseded this year [64] by experiments centred around the use of soliton crystals. These states were shown to exhibit both much higher efficiency as well as greater stability - without any requirement for closed loop feedback control systems. Moreover, newly reported states such as laser cavity solitons [133] offer even further prospects to improve both efficiency, stability and overall performance, since they do not require a CW background signal that other states (even soliton crystals) require. Very recently [68, 131, 139] there have been substantial breakthroughs in the level of integration, reliability, and low power requirements of microcombs and these breakthroughs will have direct benefits to all applications of microcombs.

Although the results reviewed here relied on some benchtop components, such as the Waveshaper, RF photonic signal processors based on Kerr micro-combs have significant potential to achieve much higher levels of integration with current nanofabrication techniques [198]. To begin with, the most important component is the micro-comb source itself, which not only is already integrated but is fabricated with CMOS compatible processes and for which there have been substantial recent developments, discussed above [131,139]. Globally established CMOS fabrication foundries can achieve advanced hybrid integration of microcomb sources and III-V devices, and this will potentially enable monolithic integration of the entire RF system. Other key components have been realized in integrated form with state-of-art nanofabrication techniques [199-201], including the optical pump source [131, 139], $\mathrm{LiNO}_{3}$ modulators [201], optical spectral shapers [199], large dispersion media [200] and photodetectors. Further, advanced integrated microcombs have been demonstrated [139] that can generate soliton crystals reliably in turn-key operation. Monolithically integrating the whole RF processing system would greatly strengthen the performance, compactness and energy efficiency of the system. Even without this, however, using the discrete integrated comb sources to replace discrete laser arrays already yields significant benefits for RF and microwave systems in terms of performance, size, cost, and complexity. Finally, soliton crystals have recently [202-205] been shown to be able to achieve a range of RF functions without the need for any spectral shaping - only by triggering the different soliton states through different pumping conditions which yields different spectra. This could potentially extend to the mid IR region as well [206-210] since these systems are not fundamentally restricted to operating in the telecom band. This adds an entirely new dimension to microcombs beyond what is possible with DKS states. This is useful since it avoids the need for spectral shapers which is an element in the RF systems that ultimately needs to be integrated.

\section{CONCLUSION}

We review recent work on the applications of Kerr micro-combs to photonic RF and microwave temporal signal processing based on transversal filters. Optical micro-combs bring a new generation of compact multi-wavelength sources to the RF photonics community and offer enormous possibilities for achieving high-performance RF signal processing with higher performance, reduced footprint, lower complexity, and potentially lower cost. By programming and shaping the comb lines according to calculated tap weights, diverse signal processing functions can be designed and have been experimentally demonstrated. We focus on fractional and integral order Hilbert transforms and differentiators as well as an RF integrator, based on integrated Kerr micro-comb sources that operate on the RF signal rather than the complex optical field. Realtime system demonstrations are also performed and show good agreement with theory. These results verify that the Kerr 
micro-comb is a highly effective tool to implement high-speed reconfigurable signal processing for future ultra-high performance RF systems.

\section{References}

[1] S. Radic, and D. J. Moss, and B. J. Eggleton, "Nonlinear optics in communications: From crippling impairment to ultrafast tools", Chapter 20, p759-828 in Optical Fiber Telecommunications V: Components and Sub-systems, Ed. Ivan P. Kaminow, Tingye Li, and Alan E. Willner, Academic Press, Oxford, UK, February 2008.

[2] J. Leuthold, C. Koos, and W. Freude, "Nonlinear silicon photonics," Nat. Photon., vol. 4, no. 8, pp. 535-544, 2010.

[3] L. Li, P. G. Patki, Y.B. Kwon, et al. “All-optical regenerator of multi-channel signals,” Nature Communications, vol. 8, Article: 884, 2017.

[4] F.Li, et al.,"All-optical XOR logic gate for 40Gb/s DPSK signals via FWM in a Si nanowire,” Opt. Express, vol.19, no.21, pp.20364-20371, 2011.

[5] F. Li, et al., "Error-free All-Optical Demultiplexing at 160Gb/s via FWM in a Si Nanowire," Optics Express, vol. 18, no. 4, pp. 3905-3910, 2010.

[6] H. Ji, et al., "1.28-Tb/s Demultiplexing of an OTDM DPSK Data Signal Using a Silicon Waveguide," Photonics Technology Letters, vol. 22, no. 23, pp. 1762-1764, 2010.

[7] C. Monat, et al., "Investigation of phase matching for third-harmonic generation in silicon slow light photonic crystal waveguides using Fourier optics," Optics Express, vol. 18, no. 7, pp. 6831-6840, 2010. DOI: 10.1364/OE.18.006831.

[8] B. Corcoran, et al., "Optical Signal Processing on a Si Chip at 640Gb/s Using Slow-Light," Optics Express, vol. 18, no. 8, pp. 7770-7781, 2010.

[9] V. G. Ta'eed, et al., "Integrated all-optical pulse regenerator in chalcogenide waveguides," Optics Letters, vol. 30, no. 21, pp. $2900-2902,2005$. DOI: $10.1364 /$ OL.30.002900.

[10] M. Rochette, et al., "Bit-error-ratio improvement with 2R optical regenerators," IEEE Phot. Technology Lett., vol. 17, no. 4, pp. 908-910, 2005.

[11] M. Ferrera, et al., "CMOS compatible integrated all-optical radio frequency spectrum analyzer," Optics Express, vol. 22, no. 18, pp. 21488 21498, 2014. DOI: 10.1364/OE.22.021488.

[12] C. Monat, et al., "Integrated optical auto-correlator based on third-harmonic generation in a silicon photonic crystal waveguide," Nature Communications, vol. 5, Article:3246, 2014. doi:10.1038/ncomms4246.

[13] F. Li, et al., "All-optical wavelength conversion for $10 \mathrm{~Gb} / \mathrm{s}$ DPSK signals in a silicon ring resonator," Optics Express, vol. 19, no. 23, pp. 2241022416, 2011.

[14] T. D. Vo, et al., "Silicon-Chip-Based Real-Time Dispersion Monitoring for 640 Gbit/s DPSK Signals," IEEE Journal of Lightwave Technology, vol. 29 , no. 12 , pp. 1790-1796, 2011.

[15] M. Ferrera, et al., "All-optical $1^{\text {st }}$ and $2^{\text {nd }}$ order integration on a chip," Optics Express, vol. 19, no. 23, pp. 23153-23161, 2011.

[16] B. Corcoran, et al., "Silicon nanowire based radio-frequency spectrum analyzer," Optics Express, vol. 18, no. 19, pp. 20190-20200, 2010.

[17] B. Corcoran, et al., "Green light emission in silicon through slow-light enhanced third-harmonic generation in photonic-crystal waveguides," Nature Photonics, vol. 3, no. 4, pp. 206-210, 2009. doi:10.1038/nphoton.2009.28.

[18] D. J. Moss, H. M. van Driel, and J. E. Sipe, "Dispersion in the anisotropy of optical third-harmonic generation in silicon," Opt. Lett., vol. 14, no. 1, pp. 57-59, 1989.

[19] J. E. Sipe, D. J. Moss, and H. M. van Driel, "Phenomenological Theory of Optical Second- And Third-Harmonic Generation Form Cubic Centrosymmetric Crystals," Phys. Rev. B, vol. 35, no. 3, pp. 1129-1141, 1987.

[20] D. J. Moss, E. Ghahramani, J. E. Sipe, and H. M. van Driel, "Band-structure calculation of dispersion and anisotropy in $\chi \rightarrow(3)$ for third-harmonic generation in Si, Ge, and GaAs," Phys. Rev. B, vol. 41, no. 3, pp. 1542-1560, 1990.

[21] D. J. Moss, H. M. van Driel, and J. E. Sipe, "Third harmonic generation as a structural diagnostic of ion implanted amorphous and crystalline silicon," Appl. Phy. Lett., vol. 48, no. 17, pp. 1150, 1986.

[22] D. J. Moss, et al., "Ultrafast all-optical modulation via two-photon absorption in silicon-insulator waveguides," Electronics Letters, vol. 41, no. 6, pp. 320-321, 2005. DOI:10.1049/el:20058051

[23] M. R. E. Lamont, et al.,"Two-photon absorption effects on self-phase-modulation-based 2R optical regeneration," Photonics Technology Letters, vol. 18 , no. 10, pp. 1185-1187, 2006. DOI:10.1109/LPT.2006.874718.

[24] A.Tuniz, G. Brawley, D. J. Moss, and B. J. Eggleton, "Two-photon absorption effects on Raman gain in single mode As2Se3 chalcogenide glass fiber," Optics Express, vol. 16, no. 22, pp. 18524-18534, 2008. DOI: 10.1364/OE.16.018524.

[25] B. J. Eggleton, B.Luther-Davies, and K. Richardson, "Chalcogenide photonics", Nature Phot., vol. 5, no. 3, pp. 141-148, 2011.

[26] M. Lee, et al., "Photosensitive post tuning of chalcogenide photonic crystal waveguides," Optics Express, vol. 15, no. 3, pp. 1277-1285, 2007.

[27] S. Tomljenovic-Hanic, M. J. Steel, C. M. d. Sterke and D. J. Moss, "High-Q cavities in photosensitive photonic crystals", Optics Letters, 32, no. 5 , pp. 542-544, 2007.

[28] C.Grillet, et al., "Nanowire coupling to photonic crystal nanocavities for single photon sources," Opt.Express, vol.15, no.3, pp.1267-1276, 2007.

[29] V. G. Ta'eed, et al., "Ultrafast all-optical chalcogenide glass photonic circuits", Opt. Express, vol. 15, no. 15, pp.9205, 2007.

[30] D. Freeman, et al., "Chalcogenide Glass Photonic Crystal Devices", Photonic and Electromagnetic Crystal Structures, Photonics and Nanostructures-Fundamentals and Applications, Science Direct Elsevier Publishing, vol. 6, no. 1, pp. 3-11, 2008.

[31] C. Grillet, et al., "Characterization and modeling of Fano resonances in chalcogenide photonic crystal membranes", Optics Express, vol. 14, no. 1 , pp. 369-376, 2006.

[32] V. G. Ta'eed, et al., "Self-phase modulation based integrated optical regeneration in chalcogenide waveguides", IEEE Journal of Selected Topics in Quantum Electronics, vol. 12, no. 3, pp. 360-370, 2006.

[33] M. Shokooh-Saremi, et al., "High performance Bragg gratings in chalcogenide rib waveguides written with a modified Sagnac interferometer: experiment and modeling", Journal of the Optical Society of America B, vol. 23, no. 7, pp. 1323-1331, 2006.

[34] M. R. E. Lamont, et al., "Error-free wavelength conversion via cross phase modulation in $5 \mathrm{~cm}^{\circ}$ of As $\mathrm{S}_{3}$ chalcogenide glass rib waveguide", Electronics Letters, vol. 43, pp. 945-947, 2007.

[35] K. Ikeda, R. E. Saperstein, N. Alic, and Y. Fainman, "Thermal and Kerr nonlinear properties of plasma-deposited silicon nitride/silicon dioxide waveguides", Opt. Express, vol. 16, pp. 12987-12994, 2008.

[36] J.S. Levy et al., "CMOS-compatible multiple-wavelength oscillator for on-chip optical interconnects," Nature Phot. vol. 4 (1) pp. $37-40$, 2010.

[37] L. Razzari, et al., "CMOS-compatible integrated optical hyper-parametric oscillator," Nature Photonics, vol. 4, no. 1, pp. 41-45, 2010.

[38] D. J. Moss, R. Morandotti, A. L. Gaeta, et al., "New CMOS-compatible platforms based on silicon nitride and Hydex for nonlinear optics," Nature Photonics, vol. 7, no. 8, pp. 597-607, 2013. 
[39] M. Ferrera, L. Razzari, D. Duchesne, et al., "Low-power continuous-wave nonlinear optics in doped silica glass integrated waveguide structures," Nature Photonics, vol. 2, no. 12, pp. 737-740, 2008.

[40] A. Pasquazi, M. Peccianti, Y. Park, B. E. Little, S. T. Chu, R. Morandotti, J. Azaña, and D. J. Moss, "Sub-picosecond phase-sensitive optical pulse characterization on a chip", Nature Photonics, vol. 5, no. 10, pp. $618-623$ (2011).

[41] D. Duchesne, M. Peccianti, M. R. E. Lamont, et al., "Supercontinuum generation in a high index doped silica glass spiral waveguide," Optics Express, vol. 18, no, 2, pp. 923-930, 2010.

[42] M. Ferrera, et al., "On-chip CMOS-compatible all-optical integrator”, Nature Communications, vol. 1, Article $29,2010$.

[43] A. Pasquazi, et al., “All-optical wavelength conversion in an integrated ring resonator,” Optics Express, vol. 18, no. 4, pp. 3858-3863, 2010.

[44] A. Pasquazi, Y. Park, J. Azana, et al., "Efficient wavelength conversion and net parametric gain via Four Wave Mixing in a high index doped silica waveguide," Optics Express, vol. 18, no. 8, pp. 7634-7641, 2010.

[45] M. Peccianti, M. Ferrera, L. Razzari, et al., "Subpicosecond optical pulse compression via an integrated nonlinear chirper," Optics Express, vol. 18 , no. 8, pp. 7625-7633, 2010.

[46] D. Duchesne, M. Ferrera, L. Razzari, et al., "Efficient self-phase modulation in low loss, high index doped silica glass integrated waveguides," Optics Express, vol. 17, no. 3, pp. 1865-1870, 2009.

[47] A. Pasquazi, M. Peccianti, L. Razzari, D. J. Moss, S. Coen, M. Erkintalo, Y. K. Chembo, T. Hansson, S. Wabnitz, P. Del'Haye, X. X. Xue, A. M. Weiner, and R. Morandotti, "Micro-combs: A novel generation of optical sources," Physics Reports, vol. 729, pp. 1-81, Jan 27. 2018.

[48] P. Del'Haye, A. Schliesser, O. Arcizet, T. Wilken, R. Holzwarth, and T. J. Kippenberg, "Optical frequency comb generation from a monolithic microresonator", Nature, vol. 450, pp. 1214-1217, 2007.

[49] M. Peccianti, et al., "Demonstration of an ultrafast nonlinear microcavity modelocked laser", Nature Communications, vol. 3, pp. 765, 2012.

[50] M. Kues, et al., "Passively modelocked laser with an ultra-narrow spectral width", Nature Photonics, vol. 11, no. 3, pp. 159, 2017.

[51] A. Pasquazi, L. Caspani, M. Peccianti, et al., "Self-locked optical parametric oscillation in a CMOS compatible microring resonator: a route to robust optical frequency comb generation on a chip," Optics Express, vol. 21, no. 11, pp. 13333-13341, 2013.

[52] A. Pasquazi, M. Peccianti, B. E. Little, et al., "Stable, dual mode, high repetition rate mode-locked laser based on a microring resonator," Optics Express, vol. 20, no. 24, pp. 27355-27362, 2012.

[53] C. Reimer, et al., "Integrated frequency comb source of heralded single photons," Optics Express, vol. 22, no. 6, pp. 6535-6546, 2014.

[54] C. Reimer, et al., "Cross-polarized photon-pair generation and bi-chromatically pumped optical parametric oscillation on a chip", Nature Communications, vol. 6, Article 8236, 2015. DOI: 10.1038/ncomms9236

[55] L. Caspani, C. Reimer, M. Kues, et al., "Multifrequency sources of quantum correlated photon pairs on-chip: a path toward integrated Quantum Frequency Combs," Nanophotonics, vol. 5, no. 2, pp. 351-362, 2016.

[56] C. Reimer et al., "Generation of multiphoton entangled quantum states by means of integrated frequency combs," Science, vol. 351, no. 6278, pp. 1176-1180, 2016.

[57] M. Kues, et al., "On-chip generation of high-dimensional entangled quantum states and their coherent control”, Nature, vol. 546, no. 7660, pp. 622-626, 2017

[58] P. Roztocki, M. Kues, C. Reimer, B. Wetzel, S. Sciara, Y. Zhang, A. Cino, B. E. Little, S. T. Chu, D. J. Moss, and R. Morandotti, "Practical system for the generation of pulsed quantum frequency combs," Optics Express, vol. 25, no. 16, pp. 18940-18949, 2017.

[59] Y. Zhang, et al., "Induced photon correlations through superposition of two four-wave mixing processes in integrated cavities", Laser and Photonics Reviews, vol. 14, no. 7, pp. 2000128, 2020. DOI: 10.1002/lpor.202000128

[60] M. Kues, C. Reimer, A. Weiner, J. Lukens, W. Munro, D. J. Moss, and R. Morandotti, "Quantum Optical Micro-combs", Nature Photonics, vol. 13, no.3, pp. 170-179, 2019.

[61] C. Reimer, et al." High-dimensional one-way quantum processing implemented on d-level cluster states", Nature Physics, vol. 15, no.2, pp. 148$153,2019$.

[62] P. Marin-Palomo, et al., "Microresonator-based solitons for massively parallel coherent optical communications", Nature, vol.546, $274,2017$.

[63] J. Pfeifle, V. Brasch, M. Lauermann, Y. Yu, D. Wegner, T. Herr, K. Hartinger, et al., "Coherent terabit communications with microresonator Kerr frequency combs", Nature Photonics, vol. 8, no. 5, pp. 375-380, 2014.

[64] B. Corcoran, et al., "Ultra-dense optical data transmission over standard fiber with a single chip source", Nature Communications, vol. 11, Article:2568, 2020.

[65] X. Xu, et al., "Photonic perceptron based on a Kerr microcomb for scalable high speed optical neural networks", Laser and Photonics Reviews, vol. 14 , no. 8,2020 . DOI:10.1002/lpor.202000070.

[66] X. Xu et al., "11 TOPs photonic convolutional accelerator for optical neural networks", Nature, vol. 589, 44-51 (2021).

[67] J. Feldmann, et al., "Parallel convolution processing using an integrated photonic tensor core", arXiv:2002.00281, 2020.

[68] D. T. Spencer, et al., “An optical-frequency synthesizer using integrated photonics”, Nature, vol. 557, no. 7703, pp. 81-85, 2018.

[69] T.Kippenberg, A.Gaeta, M.Lipson, and M.Gorodetsky, "Dissipative Kerr solitons in optical microresonators," Science, vol. 361 , no. 6402, 2018.

[70] A. L. Gaeta, M. Lipson, and T.J.Kippenberg, "Photonic-chip-based frequency combs," Nature Photonics, vol. 13, no. 3, pp. 158-169, Mar. 2019.

[71] P. Del'Haye, T. Herr, E. Gavartin, M. L. Gorodetsky, R. Holzwarth, and T. J. Kippenberg, "Octave spanning tunable frequency comb from a microresonator", Physical Review Letters, vol. 107, no. 6, pp. 063901, 2011.

[72] T. J. Kippenberg, R. Holzwarth, S.A.Diddams, "Microresonator-based optical frequency combs", Science, vol. 332, no. 6029, pp.555-559, 2011.

[73] T. Herr, V. Brasch, J. D. Jost, C. Y. Wang, N. M. Kondratiev, M. L. Gorodetsky, et al., "Temporal solitons in optical microresonators", Nature Photonics, vol. 8, no. 2, pp. 145-152, 2014.

[74] F. Ferdous, H. Miao, D. E. Leaird, K. Srinivasan, J. Wang, L. Chen, L. T. Varghese, and A. M. Weiner, "Spectral line-by-line pulse shaping of on-chip microresonator frequency combs", Nature Photonics, vol. 5, no. 12, pp. 770, 2011.

[75] X. Xue, P. H. Wang, Y. Xuan, M. Qi, and A. M. Weiner, "Microresonator Kerr frequency combs with high conversion efficiency", Laser \& Photonics Reviews, vol. 11, no. 1, 2017.

[76] X. Xue, M. Qi, and A. M. Weiner, "Normal-dispersion microresonator Kerr frequency combs", Nanophotonics, vol. 5, no. 2 , pp. $244-262$, 2016.

[77] C. Grillet, et al., "Amorphous Silicon Nanowires with Record High Nonlinearity, FOM, and Optical Stability", Optics Express, vol. 20 , no. 20. pp. 22609-22615, 2012.

[78] J. W. Choi, B. Sohn, G. F. R. Chen, D. K. T. Ng, and D. T. H. Tan, "Soliton-effect optical pulse compression in CMOS-compatible ultra-siliconrich nitride waveguides," APL Photonics, HIBSP2019, pp. 110804, 2020.

[79] J. Capmany, and D. Novak, "Microwave photonics combines two worlds," Nat. Photonics, vol. 1, no. 6, pp. 319-330, 2007. 
[80] J. P. Yao, “Microwave photonics,” Journal of Lightwave Technol., vol. 27, no. 1-4, pp. 314-335, Jan-Feb. 2009.

[81] D. Marpaung, J. Yao, and J. Capmany, “Integrated microwave photonics,” Nature Photonics, vol. 13, no. 2, 80-90, Feb. 2019.

[82] J.Azaña, “Ultrafast analog all-optical signal processors based on fiber-grating devices,” IEEE Photonics Journal, vol. 2, no. 3, pp.359-386. 2010.

[83] J. Capmany, B. Ortega, and D. Pastor, “A tutorial on microwave photonic filters,” J. Lightwave Technol., vol. 24, no. 1, pp. 201-229, 2006.

[84] V. R. Supradeepa et al., "Comb-based radiofrequency photonic filters with rapid tunability and high selectivity," Nat. Photonics, vol. 6, no. 3, pp. 186-194, Mar.2012.

[85] J. Wu, X. Xu, T. G. Nguyen, S. T. Chu, B. E. Little, R. Morandotti, A. Mitchell, and D. J. Moss, "RF Photonics: An Optical Microcombs' Perspective," IEEE J. Sel. Top. Quantum Electron., vol. 24, no. 4, pp. 6101020, Jul-Aug. 2018.

[86] V. Torres-Company, and A. M. Weiner, "Optical frequency comb technology for ultra-broadband radio-frequency photonics," Laser Photonics Rev, vol. 8, no. 3, pp. 368-393, May. 2014.

[87] Z. Jiang, C. B. Huang, D. E. Leaird, and A. M. Weiner, "Optical arbitrary waveform processing of more than 100 spectral comb lines," Nat Photonics, vol. 1, no. 8, pp. 463-467, Aug. 2007.

[88] Y. Liu, J. Hotten, A. Choudhary, B. J. Eggleton, and D. Marpaung, “All-optimized integrated RF photonic notch filter," Opt. Lett., vol. 42, no. 22, pp. 4631-4634, Nov 15. 2017.

[89] Y. Liu, D. Marpaung, A. Choudhary, J. Hotten, and B. J. Eggleton, "Link performance optimization of chip-based Si3N4 microwave photonic filters," J. Lightwave Technol., vol. 36, no. 19, pp. 4361-4370, 2018.

[90] Y. Liu, Y. Yu, S. X. Yuan, X. B. Xu, and X. L. Zhang, "Tunable megahertz bandwidth microwave photonic notch filter based on a silica microsphere cavity," Opt. Lett., vol. 41, no. 21, pp. 5078-5081, Nov 1. 2016.

[91] D. Marpaung et al., "Low-power, chip-based stimulated Brillouin scattering microwave photonic filter with ultrahigh selectivity," Optica, vol. 2, no. 2, pp. 76-83, Feb 20. 2015.

[92] A. Choudhary et al., "Advanced integrated microwave signal processing with giant on-chip Brillouin gain,” J. Lightwave Technol., vol. 35, no. 4, pp. 846-854, Feb 15. 2017.

[93] D. Marpaung, B. Morrison, R. Pant, and B. J. Eggleton, "Frequency agile microwave photonic notch filter with anomalously high stopband rejection," Opt. Lett., vol. 38, no. 21, pp. 4300-4303, Nov 1. 2013.

[94] X. Q. Zhu, F. Y. Chen, H. F. Peng, and Z. Y. Chen, "Novel programmable microwave photonic filter with arbitrary filtering shape and linear phase," Opt. Express, vol. 25, no. 8, pp. 9232-9243, Apr 17. 2017.

[95] F. Jiang, Y. Yu, H. T. Tang, L. Xu, and X. L. Zhang, "Tunable bandpass microwave photonic filter with ultrahigh stopband attenuation and skirt selectivity," Opt. Express, vol. 24, no. 16, pp. 18655-18663, Aug 8. 2016.

[96] Z. J. Zhu, H. Chi, T. Jin, S. L. Zheng, X. F. Jin, and X. M. Zhang, “All-positive-coefficient microwave photonic filter with rectangular response,” Opt. Lett., vol. 42, no. 15, pp. 3012-3015, Aug 1. 2017.

[97] G. Yu, W. Zhang, and J. A. R. Williams, "High-performance microwave transversal filter using fiber Bragg grating arrays," IEEE Photonic Tech $L$, vol. 12, no. 9, pp. 1183-1185, Sep. 2000.

[98] J. S. Leng, W. Zhang, and J. A. R. Williams, "Optimization of superstructured fiber Bragg gratings for microwave photonic filters response," IEEE Photonic Tech L, vol. 16, no. 7, pp. 1736-1738, Jul. 2004.

[99] D. B. Hunter, R. A. Minasian, and P. A. Krug, "Tunable optical transversal filter based on chirped gratings," Electron. Lett., vol. 31, no. 25, pp.2205-2207, Dec 7. 1995.

[100] E. Hamidi, D. E. Leaird, and A. M. Weiner, "Tunable Programmable Microwave Photonic Filters Based on an Optical Frequency Comb," IEEE Journal of Microwave Theory, vol. 58, no. 11, pp. 3269-3278, Nov. 2010.

[101] R. Wu, V. R. Supradeepa, C. M. Long, D. E. Leaird, and A. M. Weiner, "Generation of very flat optical frequency combs from CW lasers using cascaded intensity and phase modulators driven by tailored RF waveforms," Opt. Lett., vol.35, no.19, 3234. 2010.

[102] S. Mansoori, and A.Mitchell, "RF transversal filter using an AOTF," IEEE Photonic Tech L, vol. 16, no. 3, pp. 879-881, 2004

[103] M. Delgado-Pinar, J. Mora, A. Diez, M. V. Andres, B. Ortega, and J. Capmany, "Tunable and reconfigurable microwave filter by use of a Bragggrating-based acousto-optic superlattice modulator," Opt. Lett., vol. 30, no. 1, pp. 8-10, Jan 1. 2005.

[104] W. Z. Li, and J. P. Yao, "Optical frequency comb generation based on repeated frequency shifting using two Mach-Zehnder modulators and an asymmetric Mach-Zehnder interferometer," Opt. Express, vol. 17, no. 26, pp. 23712-23718, Dec 21. 2009.

[105] C. H. Chen, C. He, D. Zhu, R. H. Guo, F. Z. Zhang, and S. L. Pan, "Generation of a flat optical frequency comb based on a cascaded polarization modulator and phase modulator," Opt. Lett., vol. 38, no. 16, pp. 3137-3139, Aug 15. 2013.

[106] T. Saitoh, M. Kourogi, and M. Ohtsu, “An optical frequency synthesizer using a waveguide-type optical frequency comb generator at 1.5 -mu m wavelength,” IEEE Photonic Tech L, vol. 8, no. 11, pp. 1543-1545, Nov. 1996.

[107] T. G. Nguyen et al., "Integrated frequency comb source-based Hilbert transformer for wideband microwave photonic phase analysis," Opt. Express, vol. 23, no. 17, pp. 22087-22097, Aug. 2015.

[108] X. Xue, et al., "Programmable single-bandpass photonic RF filter based on a Kerr comb from a microring," Journal of Lightwave Technol., vol. 32, no. 20, pp. 3557-3565, Oct. 2014.

[109] X. Xu et al., "Reconfigurable broadband microwave photonic intensity differentiator based on an integrated optical frequency comb source," APL Photonics, vol. 2, no. 9, 096104, Sep. 2017.

[110] X. Xu, M. Tan, J. Wu, R. Morandotti, A. Mitchell, and D. J. Moss, "Microcomb-based photonic RF signal processing", IEEE Photonics Technology Letters, vol. 31 no. 23 1854-1857, 2019.

[111] X. Xu, et al., "Advanced RF and microwave functions based on an integrated optical frequency comb source," Opt. Express, vol. 26, no. 3, pp. 2569-2583, Feb. 2018.

[112] X. Xue, et al., "Microcomb-based true-time-delay network for microwave beamforming with arbitrary beam pattern control," Journal of Lightwave Technology, vol. 36, no. 12, pp. 2312-2321, Jun. 2018.

[113] X. Xu, et al., "Broadband RF channelizer based on an integrated optical frequency Kerr comb source," Journal of Lightwave Technology, vol. 36, no. 19 , pp. 4519-4526, 2018.

[114] X. Xu, et al., "Continuously tunable orthogonally polarized RF optical single sideband generator based on micro-ring resonators," Journal of Optics, vol. 20, no. 11, pp. 115701.2018.

[115] X. Xu, et al., "Orthogonally polarized RF optical single sideband generation and dual-channel equalization based on an integrated microring resonator," Journal of Lightwave Technology, vol. 36, no. 20, pp. 4808-4818. 2018. 
[116] X. Xu, et al., "Photonic microwave true time delays for phased array antennas using a 49 GHz FSR integrated optical micro-comb source," Photonics Res, vol. 6, no. 5, pp. B30-B36, 2018.

[117] X. Xu, et al., "Advanced adaptive photonic RF filters with 80 taps based on an integrated optical micro-comb source," Journal of Lightwave Technology, vol. 37, no. 4, pp. 1288-1295, 2019.

[118] W. Liang, et al., "High spectral purity Kerr frequency comb radio frequency photonic oscillator," Nature Comm., vol. 6 pp. 7957.2015.

[119] J. Liu, et al., "Photonic microwave generation in the X-and K-band using integrated soliton microcombs" Nature Phot., vol. 14, pp. 1-6, 2020.

[120] X. Xu, et al., Broadband microwave frequency conversion based on an integrated optical micro-comb source", Journal of Lightwave Technology, vol. 38 no. 2 , pp. $332-338,2020$.

[121] M. Tan, et al., "Photonic RF and microwave filters based on 49GHz and 200GHz Kerr microcombs", Optics Comm. vol. 465, 125563.2020.

[122] X. Xu, et al., "Broadband photonic RF channelizer with 90 channels based on a soliton crystal microcomb", Journal of Lightwave Technology, vol. 38, no. 18, 5116 - 5121, Sept. 15 (2020). doi: 10.1109/JLT.2020.2997699

[123] X. Xu, et al., "Photonic RF and microwave integrator with soliton crystal microcombs", IEEE Transactions on Circuits and Systems II: Express Briefs, vol. 67, no. 12, pp. 3582-3586, 2020.

[124] X.Xu, et al.,"Photonic RF phase-encoded signal generation with a microcomb source", J.Lightwave Technology, vol.38 (7) pp.1722-1727, 2020.

[125] X. Xu, et al., "High performance RF filters via bandwidth scaling with Kerr micro-combs," APL Photonics, vol. 4, no. 2, pp. 026102.2019.

[126] M. Tan, et al., "Microwave and RF photonic fractional Hilbert transformer based on a 50 GHz Kerr micro-comb", Journal of Lightwave Technology, vol. 37, no. 24, pp. $6097-6104,2019$.

[127] M. Tan, et al., "RF and microwave fractional differentiator based on photonics", IEEE Transactions on Circuits and Systems: Express Briefs, vol. 67, no.11, pp. 2767-2771, 2020.

[128] M. Tan, et al., "Photonic RF arbitrary waveform generator based on a soliton crystal micro-comb source", Journal of Lightwave Technology, vol. 38, no. 22, pp. 6221-6226, Oct 22, 2020.

[129] D. C. Cole, et al., "Soliton crystals in Kerr resonators," Nat. Photonics, vol. 11, no. 10, pp. 671-676, Oct. 2017.

[130] W. Wang et al., "Robust soliton crystals in a thermally controlled microresonator," Opt. Lett., vol. 43, no. 9, pp. 2002-2005, 2018.

[131] B. Stern et al., "Battery-operated integrated frequency comb generator", Nature, vol. 562, no. 7727, pp. 401, 2018.

[132] X. Xue, et al., "Mode-locked dark pulse Kerr combs in normal-dispersion microresonators," Nature Photonics, vol. 9, no. 9, pp. 594. 2015.

[133] H. Bao, et al., "Laser cavity-soliton microcombs," Nature Photonics, vol. 13, no. 6, pp. 384-389, Jun. 2019.

[134] X. Xue, X. Zheng, and B. Zhou, “Super-efficient temporal solitons in mutually coupled optical cavities,” Nature Photonics, May 2019.

[135] H. Zhou, et al., "Soliton bursts and deterministic dissipative Kerr soliton generation in auxiliary-assisted microcavities," Light: Science \& Applications, vol. 8, no. 1, pp. 50, 2019.

[136] H. Bao et al., "Turing patterns in a fibre laser with a nested micro-resonator: robust and controllable micro-comb generation", Physical Review Research, vol. 2, pp. 023395, 2020.

[137] L. D. Lauro, J. Li, D. J. Moss, R. Morandotti, S. T. Chu, M. Peccianti, and A. Pasquazi, "Parametric control of thermal self-pulsation in microcavities," Opt. Lett. vol. 42, no. 17, pp. 3407-3410, Aug. 2017.

[138] H. Bao, et al., "Type-II micro-comb generation in a filter-driven four wave mixing laser," Photonics Research, vol. 6, no. 5, pp. B67-B73, 2018.

[139] B. Shen, Chang, L., Liu, J., et al., "Integrated turnkey soliton microcombs," Nature, vol. 582, pp. 365-369, 2020.

[140] D. J. Moss, M. Lamont, S. Mclaughlin, G. Randall, P. Colbourne, S. Kiran and C. A. Hulse,"Tunable dispersion and dispersion slope compensators for 10Gb/s using all-pass multicavity etalons", IEEE Photonics Technology Letters, vol. 15, pp. 730, 2003.

[141] L. M. Lunardi, D. Moss, S. Chandrasekhar, L. L. Buhl, A. Hulse, P. Colbourne, G. Randall, S. Mclaughlin, “Tunable dispersion compensators based on multi-cavity all-pass etalons for 40Gb/s systems", IEEE J. Lightwave Technology, vol. 20, pp. $2136,2002$.

[142] D. J. Moss, et al., "Multichannel tunable dispersion compensation using all-pass multicavity etalons", TuT2 Optical Fiber Communications Conf., Anaheim (2002). Technical Digest (IEEE Cat. No.02CH37339). Opt Soc. America. Part vol.1, 2002, pp. 132-3. Washington, DC, USA.

[143] D. J. Moss, et al., "Tunable dispersion compensation at $10 \mathrm{~Gb} / \mathrm{s}$ and $40 \mathrm{~Gb} / \mathrm{s}$ using multicavity all-pass etalons", Optical Fiber Communications Conf. TuD1, p162, Atlanta, GA, March (2003). Digest (IEEE Cat. No.03CH37403). OSA. Part vol.1, 2003, pp. 162-3. Washington, DC, USA.

[144] S. L.Hahn, Transforms and Applications Handbook, A. D. Poularikas, Ed., 3rd ed. Boca Raton, FL: CRC Press, 2010, ch. 7.

[145] L. Moura, "Radio Frequency Implementation of the Fractional Hilbert Transform with Transversal Filters," Circuits, Systems \& Signal Processing., vol. 26, no. 3, pp. 407-417, Jun. 2007.

[146] A. Lohmann, D. Mendlovic, and Z. Zalevsky, "Fractional Hilbert transform," Opt. Lett., vol. 21, no. 4, pp.281-283, Feb. 1996.

[147] J. A. Davis et al., "Analysis of the fractional Hilbert transform," Appl. Opt., vol. 37, no. 29, pp. 6911-6913, Oct. 1998.

[148] C. D. Holdenried, J. W. Haslett, and B. Davies, "A fully integrated 10-Gb/s tapped delay Hilbert transformer for optical single sideband," IEEE Microw. Wireless Compon. Lett., vol. 15, no. 5, pp. 303-305, May 2005.

[149] H. Emami, N. Sarkhosh, L. A. Bui, and A. Mitchell, "Wideband RF photonic in-phase and quadrature-phase generation," Opt. Lett., vol. 33, no. 2, pp. 98-100, Jan 15. 2008.

[150] M. Li, and J. P. Yao, "All-fiber temporal photonic fractional Hilbert transformer based on a directly designed fiber Bragg grating," Opt. Lett., vol. 35, no. 2, pp. 223-225, Jan. 2010.

[151] M. Li, and J. P. Yao, "Experimental Demonstration of a Wideband Photonic Temporal Hilbert Transformer Based on a Single Fiber Bragg Grating," IEEE Photon. Technol. Lett., vol. 22, no. 21 pp. 1559-1561, Nov. 2010.

[152] M. H. Asghari, and J. Azana, "All-optical Hilbert transformer based on a single phase-shifted fiber Bragg grating: design and analysis," Opt. Lett., vol. 34, no. 3, pp. 334-336, Feb. 2009.

[153] T. Yang, J. Dong, L. Liu, S. Liao, S. Tan, L. Shi, D. Gao, and X. Zhang, "Experimental observation of optical differentiation and optical Hilbert transformation using a single SOI microdisk chip," Sci. Rep., vol. 4, pp. 3960, 2014.

[154] W. Liu, et al., "A fully reconfigurable photonic integrated signal processor," Nature Phot., vol. 10, no. 3, pp. 190-196, 2016.

[155] Z. Zhang, et al., "Wideband and continuously-tunable fractional photonic Hilbert transformer based on a single high-birefringence planar Bragg grating," Optics Express, vol. 26, pp. 20450-20458, 2018.

[156] F. Zeng, and J. Yao, “An Approach to Ultrawideband Pulse Generation and Distribution Over Optical Fiber," IEEE Photonics Technol. Lett., vol. 18, no. 7, pp. 823-825, Apr. 2006.

[157] S. Pan, and J. Yao, "Optical generation of polarity- and shape-switchable ultrawideband pulses using a chirped intensity modulator and a firstorder asymmetric Mach-Zehnder interferometer,” Opt. Lett., vol. 34, no. 9, pp. 1312-1314, May. 2009. 
[158] Y. Yu, J. Dong, X. Li, and X. Zhang, “Ultra-Wideband Generation Based on Cascaded Mach-Zehnder Modulators,” IEEE Photonics Technol. Lett., vol. 23, no. 23, Dec. 2011

[159] L. Zhuang, M. R. Khan, W. Beeker, A. Leinse, R. Heideman, and C. Roeloffzen, "Novel microwave photonic fractional Hilbert tra nsformer using a ring resonator-based optical all-pass filter," Opt. Exp., vol. 20, no. 24, pp. 26499-26510, Nov. 2012.

[160] C. Sima, J. C. Gates, C. Holmes, P. L. Mennea, M. N. Zervas, and P. G. R. Smith, "Terahertz bandwidth photonic Hilbert transformers based on synthesized planar Bragg grating fabrication," Opt. Lett., vol. 38, no. 17, pp. 3448-3451, Sep. 2013.

[161] H. Shahoei, P. Dumais, and J. P. Yao, "Continuously tunable photonic fractional Hilbert transformer using a high-contrast germanium-doped silica-on-silicon microring resonator,” Opt. Lett., vol. 39, no. 9, pp. 2778-2781, May 2014.

[162] Z. Li, Y. Han, H. Chi, X. Zhang, and J. P. Yao, “A continuously Tunable Microwave Fractional Hilbert Transformer Based on a Nonuniformly Spaced Photonic Microwave Delay-Line Filter,” J. Lightwave Technol., vol. 30, no. 12, pp. 1948-1953, Jun. 2012.

[163] Z. Li, H. Chi, X. Zhang, and J. P. Yao, "A continuously tunable microwave fractional Hilbert transformer based on a photonic microwave delayline filter using a polarization modulator," IEEE Photon. Technol. Lett., vol. 23, no. 22, pp. 1694-1696, 2011.

[164] A. O.-Blanch, J. Mora, J. Capmany, B. Ortega, and D. Pastor, "Tunable radio-frequency photonic filter based on an actively mode-locked fiber laser," Opt. Lett., vol. 31, no. 6, pp. 709-711, Mar. 2006.

[165] V. R. Supradeepa, C. M. Long, R. Wu. F. Ferdous, E. Hamidi, D. E. Leaird, and A. M. Weiner, "Comb-based radiofrequency photonic filters with rapid tunability and high selectivity," Nature Photonics, vol. 6, pp. 186-194, Mar. 2012.

[166] V. T.-Company, and A. M. Weiner, "Optical frequency comb technology for ultra-broadband radio-frequency photonics," Laser Photonics Rev., vol. 8, no. 3, pp. 368-393, 2014

[167] F. Li, Y. Park, and J. Azaña, "Linear Characterization of Optical Pulses With Durations Ranging From the Picosecond to the Nanosecond Regime Using Ultrafast Photonic Differentiation," J. Lightw. Technol., vol. 27, no. 1, pp. 4623-4633, 2009.

[168] S. Pan, J. Yao, "Optical generation of polarity- and shape-switchable ultrawideband pulses using a chirped intensity modulator and a first-order asymmetric Mach-Zehnder interferometer," Opt. Lett., vol. 34, no. 9, pp. 1312-1314, 2009.

[169] X. Li, J. Dong, Y. Yu, and X. Zhang, “A Tunable Microwave Photonic Filter Based on an All-Optical Differentiator,” IEEE Photon. Technol. Lett., vol. 23, no. 22, pp. 308-310, Mar. 2011.

[170] Y. Han, Z. Li, and J. Yao, “A Microwave Bandpass Differentiator Implemented Based on a Nonuniformly-Spaced Photonic Microwave DelayLine Filter," J. Lightw. Technol., vol. 29, no. 22, pp. 3470-3475, Nov. 2011.

[171] R. Ashrafi and J. Azaña, "Figure of merit for photonic differentiators," Opt. Exp., vol. 20, no. 3, pp. 2626-2639, Jan. 2012.

[172] F. Zeng and J. Yao, "Ultrawideband Impulse radio signal generation using a high-speed electrooptic phase modulator and a fiber-Bragggrating-based frequency discriminator,” IEEE Photon. Technol. Lett., vol. 18, no. 19, pp. 2062-2064, Oct. 2006.

[173] P. Li, H. Chen, M. Chen, and S. Xie, "Gigabit/s Photonic Generation, Modulation, and Transmission for a Reconfigurable Impulse Radio UWB Over Fiber System," IEEE Photon. Technol. Lett., vol. 4, no. 3, pp. 805-816, Jun. 2012

[174] Y. Yu, F. Jiang, H. Tang, L. Xu, X. Liu, J. Dong, and X. Zhang, "Reconfigurable photonic temporal differentiator based on a dual-drive MachZehnder modulator," Opt. Exp., vol. 24, no. 11, pp. 11739-11748, May 2016.

[175] P. Velanas, A. Bogris, A. Argyris, and D. Syvridis, "High-Speed All-Optical First- and Second-Order Differentiators Based on Cross-Phase Modulation in Fibers," J. Lightw. Technol., vol. 26, no. 18, pp. 3269-3276, Sep. 2008.

[176] J. Xu, X. Zhang, J. Dong, D. Liu, and D. Huang, “All-optical differentiator based on cross-gain modulation in semiconductor optical amplifier," Opt. Lett., vol. 32, no. 20, pp. 3029-3031, Oct. 2007.

[177] J. Xu, X. Zhang, J. Dong, D. Liu, and D. Huang, "High-speed all-optical differentiator based on a semiconductor optical amplifier and an optical filter," Opt. Lett., vol. 32, no. 13, pp. 1872-1874, Jul. 2007.

[178] F. Wang, J. Dong, E. Xu, and X. Zhang, "All-optical UWB generation and modulation using SOA-XPM effect and DWDM-based multichannel frequency discrimination,” Opt. Exp., vol. 18, no. 24, pp. 24588-24594, Nov. 2010.

[179] V. Moreno, M. Rius, J. Mora, M. A. Muriel, and J. Capmany, "Integrable high order UWB pulse photonic generator based on cross phase modulation in a SOA-MZI," Opt. Exp., vol. 21, no. 19, pp. 22911-22917, Sep. 2013.

[180] Q. Wang and J. Yao, "Switchable optical UWB monocycle and doublet generation using a reconfigurable photonic microwave delay-line filter," Opt. Exp., vol. 15, no. 22, pp. 14667-14672, Oct. 2007.

[181] M. Bolea, J. Mora, B. Ortega, and J. Capmany, "Optical UWB pulse generator using an $N$ tap microwave photonic filter and phase inversion adaptable to different pulse modulation formats," Opt. Exp., vol. 17, no. 7, pp. 5023-50332, Mar. 2009.

[182] B. Mathieu et al.,"Fractional differentiation for edge detection," Signal Processing, vol. 83, pp. 2421-2432, 2003.

[183] A. Oustaloup, F. Levron, B. Mathieu, and F. M. Nanot, "Frequency-Band Complex Noninteger Differentiator: Characterization and Synthesis," IEEE Trans. on Circuit and Systems - I: Fundamental Theory and Application, vol. 47, no. 1, pp. 25-39, Jan. 2000.

[184] M.-G. Suh et al., "Microresonator soliton dual-comb spectroscopy," Science, vol. 354, pp. 600-603, Nov. 2016.

[185] R. Slavik, Y. Park, M. Kulishov, R. Morandotti, and J. Azaña, "Ultrafast all-optical differentiator,” Opt. Exp., vol. 14 no, 22 , pp. 10699 , 2006.

[186] F. Liu, T. Wang, L. Qiang, T. Ye, Z. Zhang, M. Qiu, and Y. Su, "Compact optical temporal differentiator based on silicon microring," Opt. Exp., vol. 16 no. 20, pp. 15880, 2008.

[187] L. Zhang, J. Wu, X. Yin, X. Sun, P. Cao, X. Jiang, and Y. Su, “A High-Speed Second-Order Photonic Differentiator Based on Two-Stage Silicon Self-Coupled Optical Waveguide,” IEEE Photon. J., vol. 6, no. 2, pp.7900505, 2014.

[188] J. McClellan, T. W. Parks, and L. Rabiner, "A computer program for designing optimum FIR linear phase digital filters," Transactions on Audio and Electroacoustics, vol. 21, no. 6, pp. 506-526, Dec. 1973.

[189] R. C. Williamson, R. D. Esman, "RF Photonics," Journal of Lightwave Technology, vol. 26, no. 9, pp. 1145-1153, May 2008.

[190] Y. Park, T.-J. Ahn, Y. Dai, J. Yao, and J. Azaña, “All-optical temporal integration of ultrafast pulse waveforms," Optics Express, vol. 16, no. 22, pp. 17817-17825, 2008

[191] R. Slavík, Y. Park, N. Ayotte, S. Doucet, T.-J. Ahn, S. LaRochelle, and J. Azaña, "Photonic temporal integrator for all-optical computing," Optics Express, vol. 16, no. 22, pp. 18202-18214, 2008.

[192] M. H. Asghari, Y. Park, and J. Azaña, "New design for photonic temporal integration with combined high processing speed and long operation time window," Optics Express, vol. 19, no. 2, pp. 425-435, 2011

[193] W. Liu et al." "A Photonic Temporal Integrator with an Ultra-Long Integration Time Window based on an InP-InGaAsP Integrated Ring Resonator," Journal of Lightwave Technology, vol. 32, no. 20, pp. 3654-3659, 2014.

[194] Y. Park, and J. Azaña, "Ultrafast photonic intensity integrator,” Optics Letters, vol. 34, no. 8, pp. 1156-1158, 2009. 
[195] A. Malacarne, R. Ashrafi, M. Li, S. LaRochelle, J. Yao, and J. Azaña, "Single-shot photonic time-intensity integration based on a time-spectrum convolution system," Optics Letters, vol. 37, no. 8, pp. 1355-1357, 2012.

[196] J. Zhang, J.Yao, "Microwave photonic integrator based on a multichannel fiber Bragg grating," Opt. Letters, vol. 41, no. 2, pp. $273-276,2016$.

[197] M.-G. Suh, and K. J. Vahala, "Soliton microcomb range measurement," Science, vol. 359, no. 6378, pp. 884-887, 2018.

[198] V. J. Urick, "Considerations and Application Opportunities for Integrated Microwave Photonics", Paper M2B.1, IEEE/OSA Optical Fiber Communications (OFC) Conference, Anaheim, CA, 20-22 March 2016. DOI:10.1364/OFC.2016.M2B.1

[199] A. J. Metcalf, et al., "Integrated line-by-line optical pulse shaper for high-fidelity and rapidly reconfigurable RF-filtering," Optics Express, vol. 24, no. 21, pp. 23925-23940, 2016.

[200] E. Sahin et al., "Large, scalable dispersion engineering using cladding-modulated Bragg gratings on a Si chip," Applied Physics Letters, vol. 110, pp. $161113,2017$.

[201] C. Wang et al., "Integrated lithium niobate electro-optic modulators operating at CMOS-compatible voltages," Nature, vol. 562, pp.101, 2018.

[202] J. Hu et al.,"Reconfigurable radiofrequency filters based on versatile soliton microcombs", Nature Comm., vol. 11, no. 1, pp.1-9 (2020).

[203] Mengxi Tan, Xingyuan Xu, Jiayang Wu, Thach G. Nguyen, Sai T. Chu, Brent E. Little, Roberto Morandotti, Arnan Mitchell, and Da vid J. Moss, "Photonic Radio Frequency Channelizers based on Kerr Micro-combs and Integrated Micro-ring Resonators", JOSarXiv.202010.0002.

[204] Mengxi Tan, Xingyuan Xu, Jiayang Wu, Thach G. Nguyen, Sai T. Chu, Brent E. Littl, Arnan Mitchell, Roberto Morandotti, and David J. Moss, "Orthogonally polarized RF optical single sideband generation with integrated ring resonators", JOSarXiv.202010.0001.

[205] Mengxi Tan, Xingyuan Xu, Jiayang Wu, Thach G. Nguyen, Sai T. Chu, Brent E. Littl, Arnan Mitchell,Roberto Morandotti, and David J. Moss, "Orthogonally polarized RF optical single sideband generationwith integrated ring resonators", Journal of Semiconductors, 2021, In Press.

http://www.jos.ac.cn/article/shaid/4c314db6c6dcfe19c275efde2b99a22329ba658eda52233b8ab4b8a08b55a7bf.

[206] Alberto Della Torre, Milan Sinobad, Remi Armand, Barry Luther-Davies, Pan Ma, Stephen Madden, Arnan Mitchel, David J. Moss, JeanMichel Hartmann, Vincent Reboud, Jean-Marc Fedeli, Christelle Monat, Christian Grillet, accepted minor revisions, "Mid-infrared supercontinuum generation in a low-loss germanium-on-silicon waveguide", APL Photonics 6, 016102 (2021); doi: 10.1063/5.0033070.

[207] Milan Sinobad, Alberto Della Torre, Remi Armand, Barry Luther-Davies, Pan Ma, Stephen Madden, Arnan Mitchell, David J. Moss, JeanMichel Hartmann, Jean-Marc Fedeli, Christelle Monat, and Christian Grillet,"Mid-infrared supercontinuum generation in silicon-germanium all-normal dispersion waveguides", Optics Letters $\underline{45}$ (18), 5008-5011 (2020). DOI: 10.1364/OL.402159.

[208] Milan Sinobad, Alberto Della Torre, Barry Luther-Davis, Pan Ma, Stephen Madden, Arnan Mitchell, David J. Moss, Jean-Michel Hartmann, Jean-Marc Fédéli, Christelle Monat and Christian Grillet, "High coherence at fand $2 \mathrm{f}$ of a mid-infrared supercontinuum in a silicon germanium waveguide", IEEE Journal of Selected Topics in Quantum Electronics (JSTQE) 26 (2) (March/April) 8201008 (2020). Print ISSN: 1077260X, Online ISSN: 1558-4542; DOI:10.1109/JSTQE.2019.2943358.

[209] Milan Sinobad, Alberto Della Torre, Barry Luther-Davis, Pan Ma, Stephen Madden, Sukanta Debbarma, Khu Vu, David J. Moss, Arnan Mitchell, Regis Orobtchouk, Jean-Marc Fedeli, Christelle Monat, and Christian Grillet, "Dispersion trimming for mid-infrared supercontinuum generation in a hybrid chalcogenide/silicon-germanium waveguide", Journal of the Optical Society of America B (JOSA B) 36, (2) A98A104 (2019). DOI: 10.1364/JOSAB.36.000A98.

[210] Milan Sinobad, Christelle Monat, Barry Luther-Davies, Pan Ma, Stephen Madden, David J. Moss, Arnan Mitchell, David Allioux, Regis Orobtchouk, Salim Boutami, Jean-Michel Hartmann, Jean-Marc Fedeli, Christian Grillet, "High brightness mid-infrared octave spanning supercontinuum generation to 8.5 $\mu \mathrm{m}$ in chip-based Si-Ge waveguides", Optica $\underline{\mathbf{5}}$ (4) 360-366 (2018). DOI:10.1364/OPTICA.5.000360. 